\title{
Individual Phase Constitutive Properties of a TRIP-assisted QP980 Steel from a Combined Synchrotron X-ray Diffraction and Crystal Plasticity Approach
}

\author{
X.H. Hu ${ }^{\mathrm{a}}$, X. Sun ${ }^{\mathrm{a}}$, L.G. Hector, Jr. ${ }^{\mathrm{b}}$, Y. Ren ${ }^{\mathrm{c}}$ \\ ${ }^{a}$ Pacific Northwest National Laboratory, Richland, WA 99354, USA \\ ${ }^{b}$ GM R\&D, Warren, MI 48090, USA \\ ${ }^{c}$ X-ray Science Division, Argonne National Laboratory, Argonne, IL 60439, USA
}

\begin{abstract}
Microstructure-based constitutive models for multiphase steels require accurate constitutive properties of the individual phases for component forming and performance simulations. We address this requirement with a combined experimental/theoretical methodology which determines the critical resolved shear stresses and hardening parameters of the constituent phases in QP980, a TRIP assisted steel subject to a two-step quenching and partitioning heat treatment. High energy X-Ray diffraction (HEXRD) from a synchrotron source provided the average lattice strains of the ferrite, martensite, and austenite phases from the measured volume during in situ tensile deformation. The HEXRD data was then input to a computationally efficient, elastic-plastic self-consistent (EPSC) crystal plasticity model which estimated the constitutive parameters of different slip systems for the three phases via a trial-and-error approach. The EPSC-estimated parameters are then input to a finite element crystal plasticity (CPFE) model representing the QP980 tensile sample. The predicted lattice strains and global stress versus strain curves are found to be $8 \%$ lower that the EPSC model predicted values and from the HEXRD measurements, respectively. This discrepancy, which is attributed to the stiff secant assumption in the EPSC formulation, is resolved with a second step in which CPFE is used to iteratively refine the EPSC-estimated parameters. Remarkably close agreement is obtained between the theoretically-predicted and experimentally derived flow curve for the QP980 material.
\end{abstract}

Keywords: Quench and Partitioning Steels, CRSS and Hardening Parameters, Synchrotron Radiation, X-ray Diffraction, Elastic Plastic Self-Consistent Model, Crystal Plasticity Finite Element Model.

\section{Introduction}

The superior combination of strength and ductility [1] of the advanced high strength steels (AHSS) makes them particularly attractive for forming complex component geometries needed in the global automotive industries [2]. While the first [3] and second generations AHSS [4] are produced commercially, substantial effort is currently being directed toward the development of third generation AHSS (3GAHSS) with significant mechanical properties over earlier generations. Promising 3GAHSS candidates include the so-called TRIP-assisted QP (quenched and partitioned), which is produced with a quenching and partitioning (Q\&P) heat treatment process [5-7] to increase 
carbon enrichment of stability of the retained austenite for both ductility and strength enhancements [8]. Currently, QP980 produced by Bao Steel is available in the commercial domain with a nominal ultimate tensile strength (UTS) of $980 \mathrm{MPa}$.

The overwhelming impact of microstructure on the mechanical response and in-service performance of future 3GAHSS will require accurate constitutive properties of the individual phases in these materials. The fidelity of the constitutive models for component-level finite element (FE) simulations of these steels will be especially dependent upon such properties since the material response to the multiple strain paths experienced both in forming and component performance must be accurately predicted. This is the critical first step towards minimizing the number of physical tests required to validate the component-level performance of the 3GAHSS, serving the ultimate goal of accelerating the development to deployment cycle of these materials. The individual phase constitutive properties discussed here are mainly the individual phase slip system parameters of dislocations, which include the critical resolved shear stresses and hardening parameters, which will determine the plastic response of these phases during loading and ultimately the material ductility, the strength and forming behaviors of the material. They cannot be easily measured experimentally, and hence a method that combines experiment and computation is often adopted.

In-situ volumetric diffraction measurements with high energy X-ray diffraction (HEXRD) can provide experimental data for inputs to various crystal plasticity (CP) models, such as the elastoplastic self-consistent (EPSC) model [9-15] and crystal plasticity finite element (CPFE) model, with which estimation of the slip system parameters can be made. The in-situ HEXRD test generates diffraction patterns that are indicative of changes in the microstructure as a specimen is incrementally strained, such as in tensile testing [9]. For individual phases, lattice strain changes of lattice planes normal to a specific direction (e.g. the loading direction) can be calculated from the peak shifts in the recorded diffraction patterns [16].

Before straining, the texture and phase volume fractions can also be measured by HEXRD. The crystal plasticity (CP) models use the measured initial texture and phase volume fractions as inputs. With a set of assumed slip system parameters, the lattice strains as a function of macro-strains corresponding to those obtained from diffraction data can be calculated. The slip system parameters are then iteratively adjusted and a final set of parameters is obtained once the model calculated lattice strains match those from diffraction data measurement. This method of obtaining slip system parameters is hereafter referred to as the trial and error method.

In the EPSC framework, a grain aggregate of different phases, generated from the HEXRD measured texture and phase volume fraction data, is considered. Each grain is an independent Eshelby inclusion embedded in a medium with the average properties of the aggregate; hence, spatial distributions of grains as well as grain/grain and phase/phase interactions are ignored. Based on an initial set of assumed slip system parameters, the EPSC scheme computes the stress and strain state of each grain during straining. The total strain of each grain consists of elastic and plastic parts. Only the elastic strain will contribute to lattice distortion and it is used to calculate lattice strains of each grain. An average value of the lattice strains of a specific lattice plane $(h k l)$ of a specific phase $(p)$ along a specific direction, such as the LD (loading direction), $\bar{\varepsilon}_{(h k l) p}^{L D}$ (EPSC), is then calculated by averaging the lattice strains of all the grains of phase $p$ with $(h k l)$ plane normal to LD. With a trial-and-error approach, the slip system parameters for various phases are finally determined when the calculated value of $\bar{\varepsilon}_{(h k l) p}^{L D}$ (EPSC) and its evolution during deformation matches the $\bar{\varepsilon}_{(h k l) p}^{L D}$ measured with in-situ HEXRD tensile tests. 
Alternatively, finite element-based crystal plasticity (CPFE) modeling can also be used to obtain the slip system parameters of individual phases. Unlike the EPSC approach, CPFE offers a greater degree of modeling fidelity since it explicitly resolves the spatial distributions of grains with FE discretization and considers the grain/grain and phase/phase interactions thereby accurately capturing the instantaneous compatible response among various grains and phases during plastic deformation [17, 18]. By explicitly resolving different grain orientations and phases in the FE meshes, however, each CPFE-based simulation run requires orders of magnitude higher computational time than that of an EPSC calculation, and the trial-and-error approach in identifying the individual phase properties may become computationally prohibitive for multiphase steels solely with CPFE modeling. Most of the literature-reported HEXRD-based phase property determination works use EPSC-based approach. Also note that EPSC analyses reported in the literature have focused primarily on the first generation AHSS, typically excluding austenite because of the low volume fraction $(<5 \%)[10,11]$. As noted by Hu et al. [16], the austenite phase in the 3GAHSS should be considered in CP models since the volume fraction can well exceed 5\%. However, the complex behavior associated with strain-induced austenite to martensite phase transformation has not been directly considered in any crystal plasticity finite element modeling in calculating lattice strains due to the nature of non-continuity in the transformation.

The average stress vs strain relationships for individual phases can also be obtained with CP models (EPSC or CPFE) with the appropriate set of slip system parameters for each phase. The final computed set, which meet the aforementioned criteria, can subsequently be used in the microstructure-based property predictions [28, 32] or in state variable-based constitutive models for forming and component-level performance predictions [19].

At the present time, no study in the literature has compared the results of the EPSC calculated average phase properties such as flow stresses to other CP methods, such as CPFE models with the EPSC-estimated phase slip system parameters for multi-phase steels. The present study aims to fill this gap. We focus on determining the slip system parameters as well as average stress vs strain relationships for individual phases of QP980, a commercial, multi-phase (ferrite (BCC), martensite (BCT), austenite (FCC), and new martensite (BCT)) 3GAHSS. We initially use the computationally efficient EPSC model to determine the individual phase constitutive parameters with a trialand-error approach. These parameters are then input to a CPFE model of a polycrystalline virtual tensile sample. The results indicate that the CPFE predicted macroscopic stress vs. strain curve with the EPSC-generated phase parameters is consistently $\sim 8 \%$ lower than that obtained from EPSC and $\sim 10.5 \%$ lower than the experimental results. The CPFE calculated lattice strains are also found to be lower in three of the phases. To obtain more accurate slip system parameters associated with each phase in the material, a $2^{\text {nd }}$ step involving CPFE-based HEXRD data interpretation is used to further refine the EPSC-calculated initial values of slip system parameters for individual phases. With the CPFE-refined parameter set for individual phases, good comparison has been achieved between the predicted and experimentally measured lattice strains for various phases and the macroscopic stress versus strain curve for the QP980. In addition, the CPFE-based model can also capture the lattice strain fluctuations observed in the HEXRD data. Note that phase transformation is not directly considered in all three crystal plasticity models (EPSC, CPFE). Rather, $50 \%$ of the retained austenite is assumed to have transformed to new martensite at the outset of deformation, while the remaining $50 \%$ austenite is untransformed in the microstructure throughout the deformation process. While the obtained phase properties for retained austenite and new martensite are considered not as accurate as those for ferrite and tempered martensite, we show that such an assumption yields reasonable 
results in terms of predicted lattice strains and macroscopic stress vs. strain relationships for the current Q\&P steel with $\sim 12 \%$ retained austenite.

The remainder of this paper is organized as follows. The QP980 material is described in Section 2. In Section 3, the HEXRD experiments and analyses of the HEXRD data to obtain phase texture, volume fractions and lattice strains are detailed. In section 4, with HEXRD results, EPSC is used to obtain the slip system parameters. Then the EPSC calculated average phase stresses and macroscopic stresses are compared with those calculated by CPFE model using the same set of parameters. In section 5, CPFE model is used to refine those parameters for improved accuracy and average phase stress vs strain relationships are determined.

\section{Material}

A commercial 3GAHSS QP980 (BAO Steel), which is a TRIP-assisted steel with $~ 11.5 \%$ retained austenite, is of focus in this study. The material was produced by a Q\&P process [5-7]. The as-received microstructure consists mainly of ferrite $(\alpha)$, tempered martensite $\left(\alpha^{\prime}\right)_{1}$ and retained austenite $(\gamma)$. The martensitic phase, formed after quenching, has a BCT structure with a unit cell that is a distortion of the BCC ferrite phase, a consequence of the anisotropic $\mathrm{C}$ distribution. The reduction of carbon in the martensite matrix during partitioning alters the lattice parameter of martensite to make the BCT crystal structure closer to the cubic ferrite, the equilibrium phase at the partitioning temperature. These changes render the martensitic phase softer after partitioning relative to the martensite after quenching. The martensite after partitioning is thus tempered martensite, hereafter denoted as $\alpha_{\mathrm{T}}^{\prime}$. The QP steel considered here has an ultimate tensile strength of $980 \mathrm{MPa}$, with chemical composition listed in Table 1 .

Table 1. Chemical composition of QP980

\begin{tabular}{|c|c|c|c|c|c|c|}
\hline Element & $\mathrm{C}$ & $\mathrm{Si}$ & $\mathrm{Mn}$ & $\mathrm{P}$ & $\mathrm{S}$ & $\mathrm{Al}$ \\
\hline Weight percent, \% & 0.2 & 1.49 & 1.82 & 0.017 & 0.0043 & 0.046 \\
\hline
\end{tabular}

Note that $\mathrm{Si}$ is added to delay carbide formation, increasing the hardenability of austenite, while Mn is an austenite stabilizer. Fig. 1(a) is an optical micrograph (OM) and Fig. 1(b) is a field emission gun scanning electron microscope (FEG-SEM) micrograph of the QP980 microstructure. These show the three major constituent phases: ferrite $(\alpha)$, tempered martensite $\left(\alpha_{\mathrm{T}}^{\prime}\right)$ and retained austenite $(\gamma)$ in both blocky $\left(\gamma_{\mathrm{B}}\right)$ and lath form $\left(\gamma_{\mathrm{L}}\right)$. The lath austenite and tempered martensite are not clearly discernible in the optical micrograph (Fig. 1(a)) due to low resolution, and the entire colony (tempered martensite $\left(\alpha_{T}^{\prime}\right)$ and lath austenite $\left(\gamma_{\mathrm{L}}\right)$ ) is designated as "MA constituent" in the literature [20]. According to Xiong et al. [21], the austenite phase in QP980 appears at two disparate length scales: blocky (micron-sized grains) and film-like (lath form with sub-micron-sized grains). They noted that the larger, higher $\mathrm{C}$ content blocky austenite transforms to twinned martensite at $2 \%$ strain and is completely consumed at $12 \%$ tensile strain. However, most of the smaller and lower C content film-like austenite had yet to transform at $12 \%$ tensile strain. 


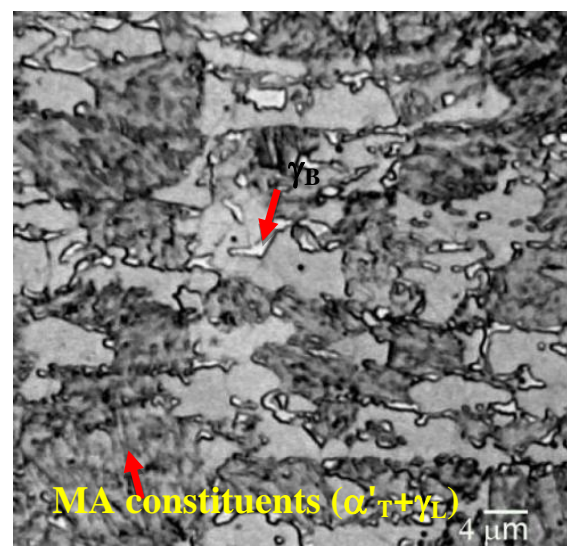

(a) $\mathrm{OM}$

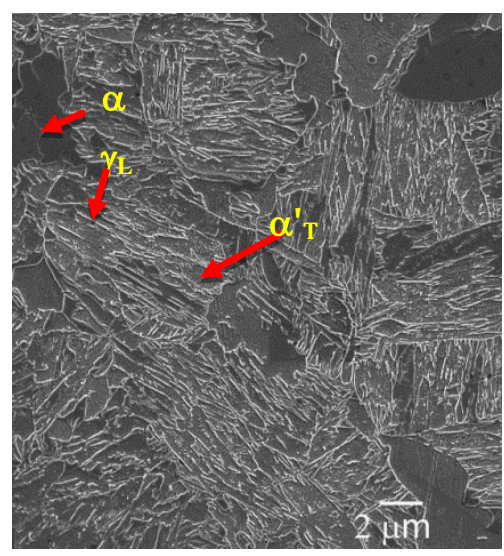

(b) FEG SEM

Fig.1 The microstructure of the QP980 steel is shown in: (a) an optical micrograph (OM) and (b) FEG-SEM image, where $\boldsymbol{\alpha}_{\mathbf{T}}{ }_{\mathrm{T}}$ is tempered martensite, $\alpha$ is ferrite, $\gamma_{B}$ is retained austenite in blocky form and $\gamma_{L}$ is retained austenite in lath (or film) form, MA constituent denotes tempered martensite $\left(\boldsymbol{\alpha}_{\mathrm{T}}^{\prime}\right)$ and lath austenite $\left(\gamma_{\mathrm{L}}\right)$.

\section{Experimental Details and Results}

\section{1 in-situ HEXRD tensile test and flow curves}

Fig. 2(a) is a schematic illustration of the in-situ synchrotron-based HEXRD tensile test setup at beam line 11ID-C of the Advanced Photon Source (APS) at Argonne National Laboratory. The synchrotron beam energy and wavelength $(\lambda)$ are $106.42 \mathrm{keV}$ and $0.01165 \mathrm{~nm}$, respectively. Sub-sized dog-bone shaped sheet samples, with $\sim 1.0$ $\mathrm{mm}$ thickness, were used. During a test, a monochromatic synchrotron X-ray beam impinges the center of the sample gauge section. The incident beam is nearly square, $(500 \mu \mathrm{m} \times 500 \mu \mathrm{m})$ and will diffract as it penetrates through the crystal aggregates of the entire QP980 thickness according to Bragg's law [22]:

$$
2 d_{h k l} \sin \theta_{h k l}=\lambda
$$

where $h k l$ denote the Miller indices of the lattice planes, and $d_{h k l}$ and $\theta_{h k l}$ are the spacing and diffraction angle for the $(\mathrm{hkl})$ planes, respectively. The diffracted X-ray beam from the lattice planes of a randomly textured polycrystalline material forms a series of cones each of which is associated with a specific lattice plane. A two dimensional Perkin-Elmer $\alpha \mathrm{Si}$ flat panel detector lies at distance D behind the sample and captures the diffracted beams as circular Debye rings for different lattice planes.

Fig. 2(b) illustrates the tensile sample geometry, with $10 \mathrm{~mm}$ gage length. The custom-designed tensile load frame has a $13 \mathrm{kN}$ capacity. The distance $\mathrm{D}$ is $1.896 \mathrm{~m}$ and is calibrated via diffraction of a standard $\mathrm{CeO}_{2}$ sample. The tensile test was conducted in displacement-controlled mode at a constant cross-head speed of $30 \mu \mathrm{m} / \mathrm{s}$. After each displacement increment of $40 \mu \mathrm{m}$, the image on the area detector was recorded by a digital camera with 10-one second exposures. The sample was positioned such that one of the planar surfaces (LD $\times \mathrm{TD}$ ) was normal to the incident beam during the in-situ tensile test. Here, LD is the loading direction (the rolling direction in the current study), while TD and ND are the transverse and normal directions, respectively. 
Before the tensile test, diffraction patterns were also recorded as the sample was rotated around the loading direction (LD) from 0 to $90^{\circ}$ to obtain the initial texture of the material. The HEXRD-measured texture is volumetric and all grains through the thickness of a tensile sample $(\sim 1 \mathrm{~mm})$ that fall within the beam contribute to the measured diffraction patterns. This gives a much better statistical measurement of the material texture relative to $\mathrm{Cu} \mathrm{K} \alpha \mathrm{X}$-ray diffraction which has lower energy and operates in the reflection mode, as shown in the literature [23], where penetration depth is only in the range of several microns.

Measured Debye rings before loading ( $\varepsilon=0$, where $\varepsilon$ denotes strain) of the QP980 steel for various diffracting planes of the austenite $(\gamma)$ and ferrite-like phases $(\alpha)$ are shown in the images in Fig. 3(a).

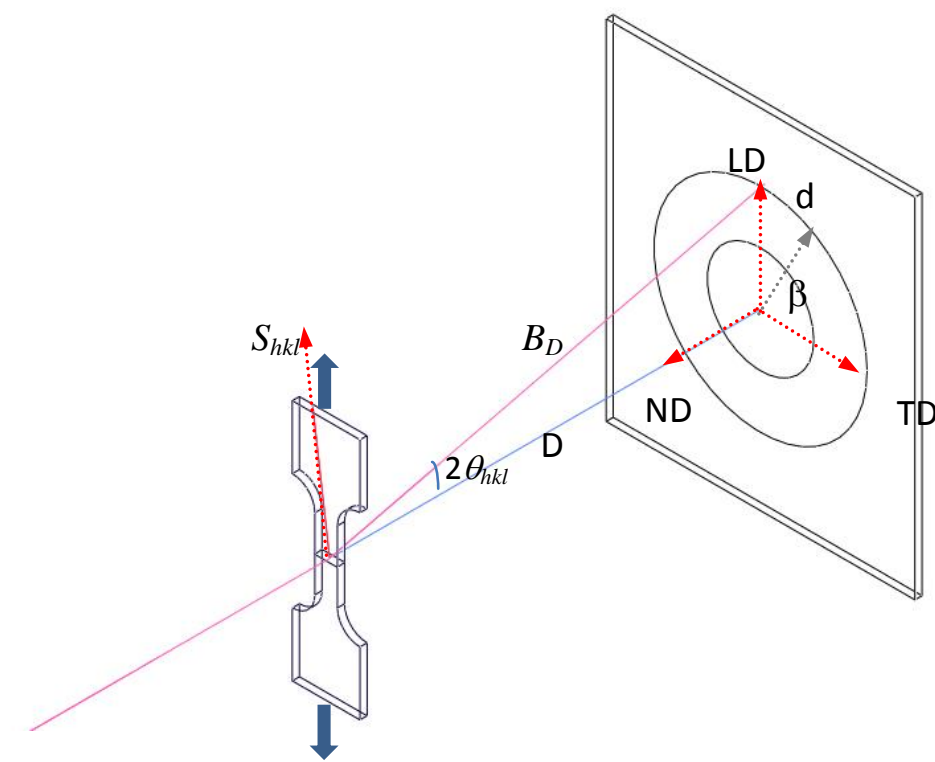

$B_{I}$ (a)

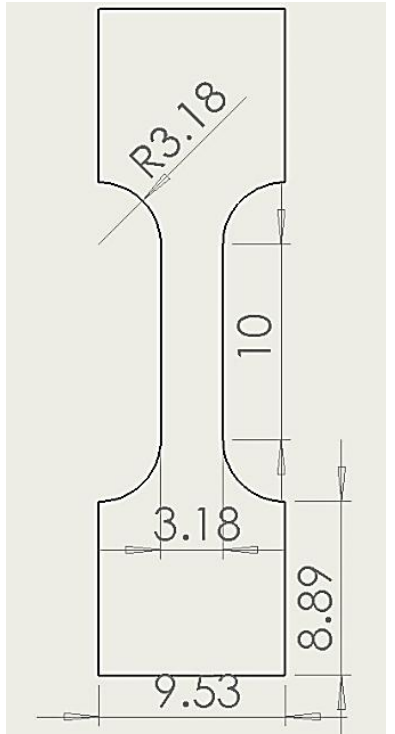

(b)

Fig. 2 (a) Schematic of in-situ synchrotron-based HEXRD tensile test setup, where $B_{I}$ and $B_{D}$ are incident and diffracted beams, and $S_{h k l}$ is the diffraction vector; (b) tensile test sample geometry, all dimensions in $\mathrm{mm}$. Note: TD = transverse direction, LD = loading direction (tensile axis) and $\mathrm{ND}=$ normal direction. $\mathrm{D}$ is the distance from sample to the area detector and $\mathrm{d}$ is the distance of a vector from the center of the Debye ring.

The load cell attached to the tensile test frame generates an analog electric voltage signal which is recorded by a computer controller, and the obtained voltage data is used to calculate the actual load applied to the frame and the engineering stress of the sample. Load frame displacement data is used to calculate the engineering strain of the sample during the tensile test. Because of the high stiffness of the QP980 sample relative to the load frame, the stiffness correction procedure from $\mathrm{Hu}$ et al. [43] is applied to the engineering stress and strain calculations to obtain the true stress versus strain curves for three QP980 samples, as shown in Fig. 4(a). The stiffness correction routine gives an accurate value of the engineering strain, which correlates very well with recent in-situ measurement results from digital image correlation (DIC) [24]. Figure 4 also shows good test repeatability for the three QP980 samples. The $\sigma_{0.2}$ yield stress is $\sim 750 \mathrm{MPa}$, the ultimate tensile strength (UTS) is $990 \mathrm{MPa}$ and the total elongation is $\sim 25 \%$. The $\sigma_{0.2}$ and UTS results are quite close to the baseline QP980 steel reported by Coryell et al. [23]. The total 
elongation is larger, however, which is typical for a sub-sized sample with small gauge length/width ratio in comparison with a standard ASTM sample. The resulting flow data will be used to validate the crystal plasticity model predictions of the macroscopic-scale flow behavior in the subsequent sections.

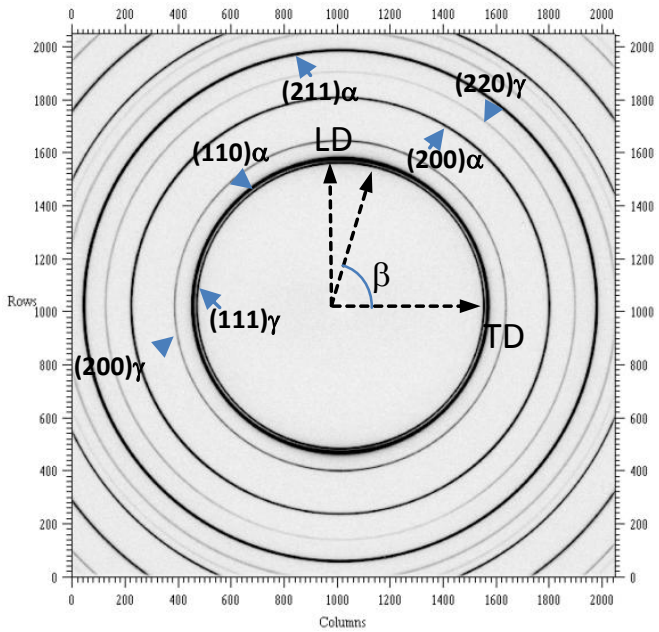

(a)

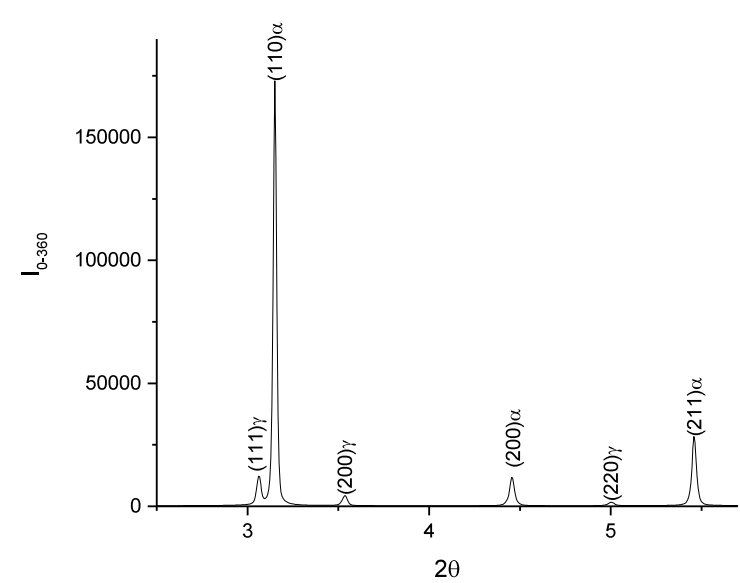

(b)

Fig. 3 Debye rings recorded from a QP980 tensile sample and the integrated intensity of the rings from $\beta=0$ to $360^{\circ}$ as a function of the $2 \theta$ angle before loading ( $\varepsilon=0)$ (a) and (b)

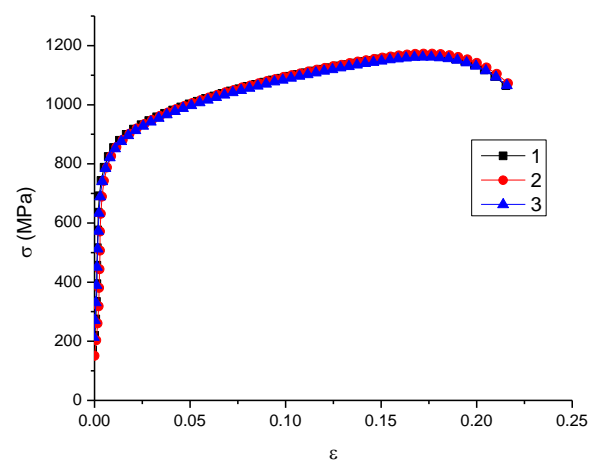

(a)

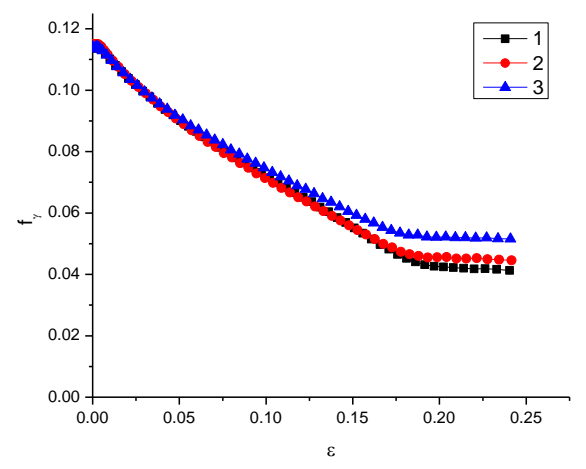

(b)

Fig. 4. (a) macroscopic-true stresses $(\sigma)$ and (b) retained austenite volume fraction $\left(f_{\gamma}\right)$ as a function of macro-true strain $(\varepsilon)$ from in situ tensile tests of three-QP980 samples (denoted as 1, 2 and 3) using the load frame at beam line 11-ID-C of APS. The macro-stresses and strains were stiffness-corrected and each sample was strained to fracture.

\subsection{HEXRD data processing}

\subsubsection{Phase volume fractions and texture}

\subsubsection{Austenite volume fraction}

Data from the recorded 2D HEXRD Debye ring images was first processed by the X-ray analysis software fit2D [25]. Firstly, the intensity of an image was integrated over $\beta$ (see Fig. 3(a)). Given that $\beta$ ranges from $0 \sim 360^{\circ}$, the integrated intensity, $I_{0-360}$, can then be calculated relative to the $2 \theta$ angle. The resulting $I_{0-360}-2 \theta$ profiles are shown in Figs. 3(b), which can be used to calculate phase volume fractions. Note that the profiles extend up to $2 \theta \sim$ 
$5.7^{\circ}$, beyond which only partial Debye rings are recorded. The OriginPro software [26] was used to fit the diffraction peaks of the various phases, and the area under a given peak, $A_{i}^{j}$, was obtained for the lattice plane $i$ of phase $j$. The volume fraction of phase $j$ was calculated with the following equation [27]:

$f^{j}=\frac{1}{P_{j}} \sum_{i=1}^{P_{j}}\left(A_{i}^{j} / R_{i}^{j}\right) \cdot\left(\sum_{k=1}^{P h}\left(\frac{1}{P_{k}} \sum_{i=1}^{P_{k}} A_{i}^{k} / R_{i}^{k}\right)\right)^{-1}$

Here, $P h$ denotes the number of phases present, $P_{j}$ accounts for the number of peaks of phase $j$ used in the calculation, and $R_{i}^{j}$ is the X-ray normalization factor for peak $i$ in phase $j$, which can be theoretically calculated $[28,29]$. In QP980 steel, three constituent phases, namely, austenite $(\gamma$, FCC), ferrite $(\alpha$, BCC), and tempered martensite $\left(\alpha_{\mathrm{T}}^{\prime}, \mathrm{BCT}\right)$ comprise the as-received microstructure. One additional phase, new martensite $\left(\alpha_{\mathrm{N}}^{\prime}, \mathrm{BCT}\right)$, forms during tensile deformation. Since the BCT martensite has a crystal structure that is similar to BCC ferrite (but with slightly different lattice parameters), the peaks of these phases overlap in the diffraction rings and the integrated diffraction profiles. Hence, the peaks of $\alpha, \alpha_{T}^{\prime}$, and $\alpha_{n}^{\prime}$ are not explicitly differentiated in the calculation of austenite $(\gamma)$ volume fraction, and we refer to them collectively as ferrite-like phases $\left(\alpha+\alpha^{\prime}{ }_{T}+\alpha^{\prime}{ }_{N}\right)$ or simply $\alpha$-like phases in Fig. 3. In Fig. 3(b), the first three peaks (at small $2 \theta$ ) associated with the austenite phase are (111), (200) and (220). Note that the measurement cannot distinguish between blocky and film austenite. The first three peaks associated with the ferrite-like phases are (110), (200) and (211). The calculated austenite volume fraction, $\mathrm{f}_{\gamma}$, with macroscopic tensile strain, $\varepsilon$, is shown as Fig. 5 for the three samples tested. Here, the initial $\mathrm{f}_{\gamma}$ is $\sim 0.115$ and it decreases to $0.04 \sim 0.05$ at the end of deformation. Note that the retained austenite volume fraction variation with strain is only for reference here and will not be considered in the crystal plasticity models (EPSC or CPFE), where phase transformation is neglected. Rather, $50 \%$ of the austenite is assumed to have transformed into new martensite at the outset of deformation. The initial $\mathrm{f}_{\gamma}$ is slightly higher than the $\sim 0.095$ value reported by Coryell et al. [23] from $\mathrm{Cu} \mathrm{K} \alpha \mathrm{XRD}$, but very close to the 12\% HEXRD result in Xiong et al. [21] for similar QP980 material from Bao steel. It is noted that the $50 \%$ transformation assumption is rather subjective, although it is better than the assumption of no austenite in the system at all like in similar works. As will be discussed later, this assumption will cause more errors on calculation results of austenite and new martensite.

\subsubsection{Ferrite and tempered martensite volume fraction}

The initial ferrite $(\alpha)$ and tempered martensite $\left(\alpha_{T}^{\prime}\right)$ volume fractions are estimated using the areas of the two Gaussian peaks, corresponding to the two ferrite-like phases before deformation. The two Gaussian peaks are labeled "Fit Peak 1 for $\alpha_{T}^{\prime}$ " and "Fit Peak 2 for $\alpha$ ". Based on the areas of the Gaussian peaks for ferrite and martensite, the volume fraction of tempered martensite is estimated to be $\sim 58.4 \%$ among the ferrite-like phases. Considering the initial $\sim 11.5 \%$ of retained austenite, the initial volume fractions of tempered martensite and ferrite are estimated to be $51.7 \%$ and $36.8 \%$, respectively. The volume fractions of the various phases are actually very close to the reported values obtained from image analysis of QP980 by Coryell et al. [23]. 


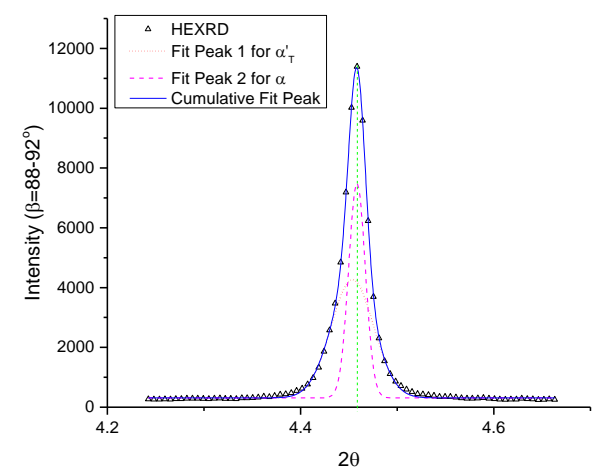

Fig. 5. The asymmetric overlapped 200 peak before deformation of the ferrite-like phases are fitted with two Gaussian peaks to calculate the initial volume fraction of the ferrite and tempered martensite.

\subsubsection{Initial textures of austenite and ferrite-like phases}

As mentioned previously, initial textures of ferrite-like phases and austenite will be used as input to the EPSC and CPFE crystal plasticity models. To measure the initial texture of the QP980 sample, the Debye rings were acquired incrementally by rotating the tensile sample around the LD from 0 to $90^{\circ}$ relative to the normal direction (ND) at $4^{\circ}$ increments. The pole figures for different lattice planes of the austenite $(111,200$ and 220) and ferritelike phases $(110,200$ and 211) were then calculated from those Debye rings with a novel correction method, where the intensity of each measurement point is normalized by the integrated value of the full recorded image. This method not only corrected the effects due to changing X-ray penetration thickness of the sample when rotating, but also the intensity variations of incident beam. This method will be discussed in more detail in another paper [30]. These corrected pole figures are then analyzed with MTM-FHM, a texture analysis software package developed by Van Houtte [31], from which the orientation distribution function (ODF) is obtained and the various pole figures for austenite and ferrite-like phases recalculated. The recalculated pole figures are plotted in Fig. $6(\mathrm{a})-(\mathrm{b})$. The $\phi_{2}=45^{\circ}$ sections of the orientation distribution function (ODF) in Euler space for both the ferrite-like phases and the austenite phase are shown in Fig. 6(c)-(d). Note that $\phi_{2}$ is the third Euler angle which represents the crystal orientation of grains $\left(\mathrm{g}=\left(\phi_{1} \psi \phi_{2}\right)\right)$ in Bunge's notation [32] . Both the ferrite-like phases and the austenite phase have a rolling texture that is commonly observed for BCC and FCC alloys [33].

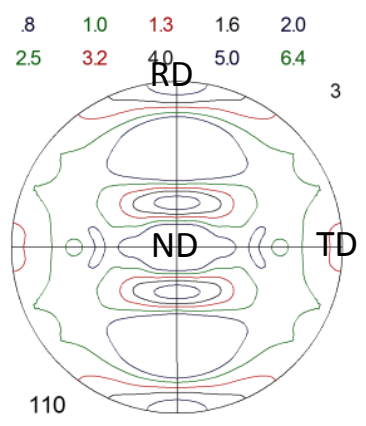

(a) $(110)_{\alpha} \mathrm{PF}$

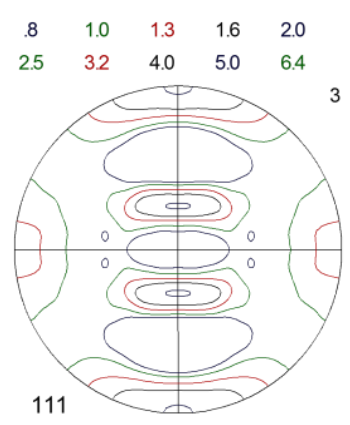

(b) $(111)_{\gamma} \mathrm{PF}$

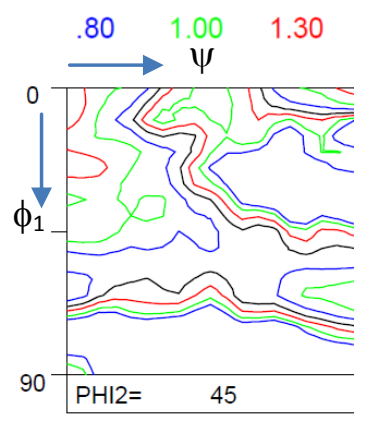

(c) $\phi_{2}=45^{\circ}$ section ODF of $\alpha$

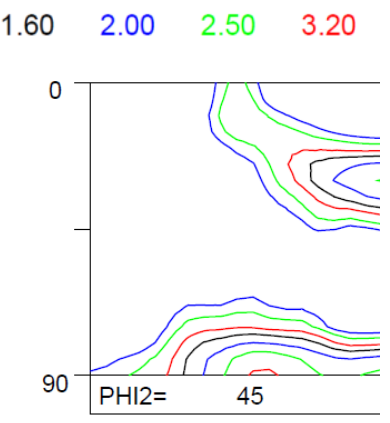

(d) $\phi_{2}=45^{\circ}$ section ODF of $\gamma$

Fig. 6. The pole figures (PF) and orientation distribution function (ODF) for the following planes/phases measured before deformation of a QP980 tensile sample: (a) (110) PF for ferrite-like phases; (b) (111) PF for austenite; the $\phi_{2}=45^{\circ}$ section of the ODF of (c) ferrite-like phases and (d) austenite, where $\phi_{1}, \psi$ and $\phi_{2}$ are the Euler angles in Bunge's Notation [32]. At the top of the figures are the contour levels of the distribution functions. 


\subsubsection{Calculation of the lattice strains}

When the sample is under tensile load, the lattice of each crystallite (e.g. grains) will stretch along the LD and shrink along TD. The lattice strains along the LD or the TD can be calculated from the shifts of lattice plane spacing $\left(d_{h k l}\right)$ or diffraction angles $\left(\theta_{h k l}\right)$. For the calculation of lattice strains along the $\mathrm{LD}$, the integration along the arc with $\beta=88-92^{\circ}$ (see Fig. 3(a)) is performed and the $I_{88-92}-2 \theta$ curves can then be obtained from fit2D for each increment of deformation. The diffraction vector of those planes with $\beta=88-92^{\circ}$ has a very small angular deviation with respect to the LD. The (111) $\gamma$ and (110) $\alpha$ peaks are too close to each other and will gradually overlap with increasing strain; therefore, the peak shifts and lattice strains of those planes are not further considered. Hence, the lattice strains for the (200) and (220) planes of the austenite and those for the (200 and (211) planes of the ferrite-like phases will be calculated. Note that all of the analyses discussed hereinafter are focused on sample 1 (results from the other samples showed negligible differences).

\subsubsection{Austenite Lattice strains}

Figure 7(a) shows the (200) $\gamma$ peaks before and after loading $(\varepsilon=0.14)$ of the QP980 steel where peak shifting from tensile straining is observed. Gaussian distribution functions are used for peak fitting, denoted as 'Gauss Fitting' in Fig. 9, where the diffraction angle before $\left(2 \theta_{h k l}^{0}\right)$ and after deformation $\left(2 \theta_{h k l}^{t}\right)$ can be determined. Here, the superscript represents time, with 0 denoting before deformation and $t$ denoting the current deformation time. In the literature [10], the engineering strain formulation is usually adopted to calculate lattice strains from the changes of lattice spacing or diffraction angle via

$$
\varepsilon_{h k l}^{e n g}=\frac{\left(d_{h k l}^{t}-d_{h k l}^{0}\right)}{d_{h k l}^{0}}=\frac{\sin \theta_{h k l}^{0}}{\sin \theta_{h k l}^{t}}-1
$$

The (200) and (220) lattice strains calculated for austenite are shown in Fig. 7(b). Here, the (200) lattice strain for austenite along the LD is much higher than that of the (220) planes. Further, the lattice strain of the (200) increases much faster than that of the (220) lattice planes due to deformation-induced anisotropy (to be discussed shortly).
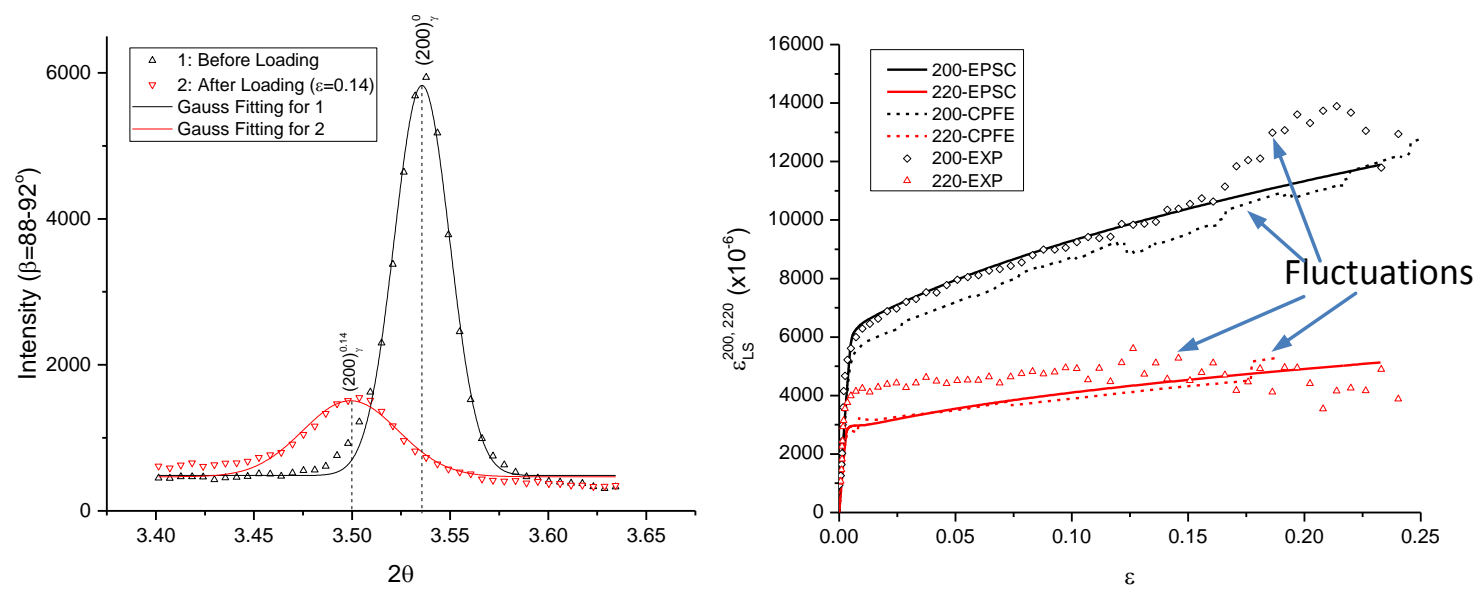

(a)

(b) 
Fig. 7. (a) The integrated intensity (around the LD: $\beta=88-92^{\circ}$ ) vs. $2 \theta$ curves show the shifting of the (200) $\gamma$ peaks before and after loading for QP980 sample 1. (b). Austenite lattice strains (in reference of macroscopic strains) of grains whose (200) or (220) plane normals parallel to the LD obtained from different sources: HEXRD data (discrete points) and calculated from CP models (EPSC (solid lines) and CPFE (dashed lines)). The CP model uses slip system parameters shown in Table 2.

\subsubsection{Peak separation and lattice strains of the ferrite-like phases}

Due to similar crystal structures, the diffraction peaks for the various ferrite-like phases overlap with each other. To determine the lattice strain of a lattice plane of each ferrite-like phase $\left(\alpha, \alpha_{\mathrm{T}}^{\prime}\right.$ or $\left.\alpha_{\mathrm{N}}^{\prime}\right)$, the overlapping peaks for the planes of the ferrite-like phases, such as (200) and (211), must be de-convoluted. This is accomplished with Gaussian peak fitting using the OriginPro analysis program [26].

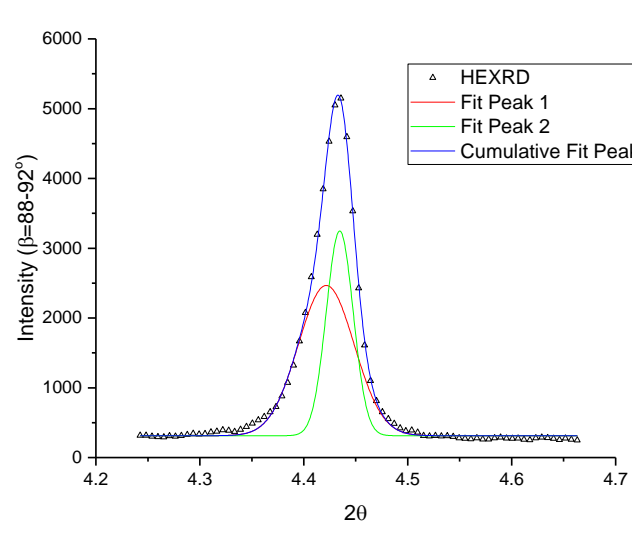

(a) $\varepsilon=0.14$, two peak fitting

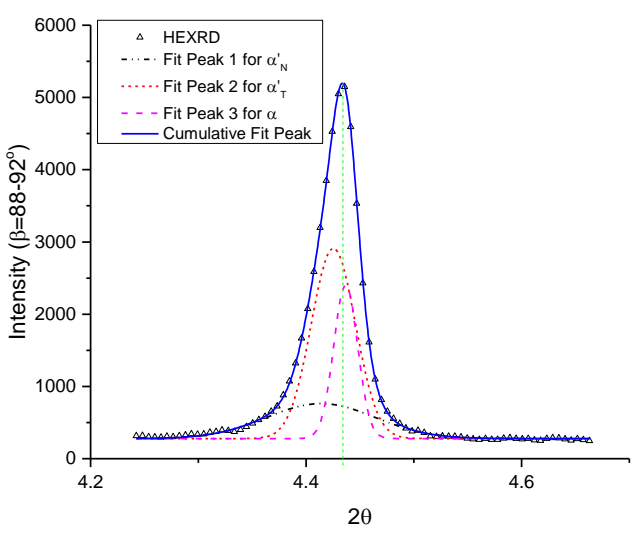

(b) $\varepsilon=0.14$, three peak fitting

Fig. 8. (200) lattice plane peak fitting of HEXRD profile of the ferrite-like phases: $\alpha$ (ferrite), $\alpha^{\prime}{ }_{\mathrm{T}}$ (tempered martensite) and $\alpha^{\prime}{ }_{\mathrm{N}}$ (new martensite), at $\varepsilon=0.14$ : (a) two peak fitting and (b) $\varepsilon=0.14$, three peak fitting of QP980 steel sample 1 .

The discrete symbols in Fig. 5 show the HEXRD diffraction pattern for the (200) lattice plane of the ferrite-like phases before deformation. The clear asymmetry between the right and left portion of the measured diffraction pattern (separated by the green dashed line in Fig. 5) indicates that two Gaussian peaks can yield a good fit to the measured diffraction peak (see Fig. 5). Under a deformed condition, however, the (200) diffraction pattern becomes more and more asymmetric (see Fig. 8(a)). This is due to the different deformation behaviors among the two original phases $\left(\alpha\right.$ and $\left.\alpha_{T}^{\prime}\right)$ and the introduction of the freshly formed martensite, $\alpha^{\prime}{ }_{N}$, via austenite transformation. In this case, two Gaussian peaks will no longer yield satisfactory fitting of the asymmetric diffraction pattern, particularly for the regions with $2 \theta$ around $4.35^{\circ}$, see Fig. 8(a), indicating that $\alpha^{\prime}{ }_{N}$ needs to be considered in the diffraction peak separation for the ferrite-like phases under a deformed condition. Fig. 8(b) shows a good three peak fitting considering the $\alpha^{\prime}{ }_{N}$ resulted from austenite transformation $\left(\gamma \rightarrow \alpha^{\prime}{ }_{N}\right)$ at $\varepsilon=0.14$.

After peak separation, the $2 \theta_{h k l}(h k l=200$ or 211) angles for the various ferrite-like phases are obtained. The lattice strains can then be calculated with Eq. (3), where $\theta_{h k l}^{0}$ is taken as the value obtained at zero strain. Since $\alpha_{N}^{\prime}$ does not exist prior to straining, its $\theta_{h k l}^{0}$ value is assumed to be that of $\alpha_{\mathrm{T}}^{\prime}$. The lattice strains for the (200) and (211) planes thus obtained for the ferrite-like phases as a function of macro-strain are shown in Fig. 9 (a) and (b) as open symbols. 


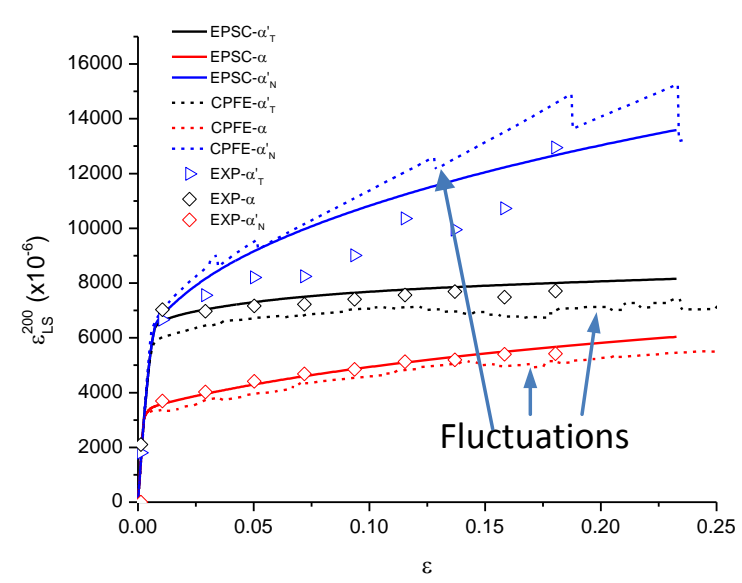

(a)

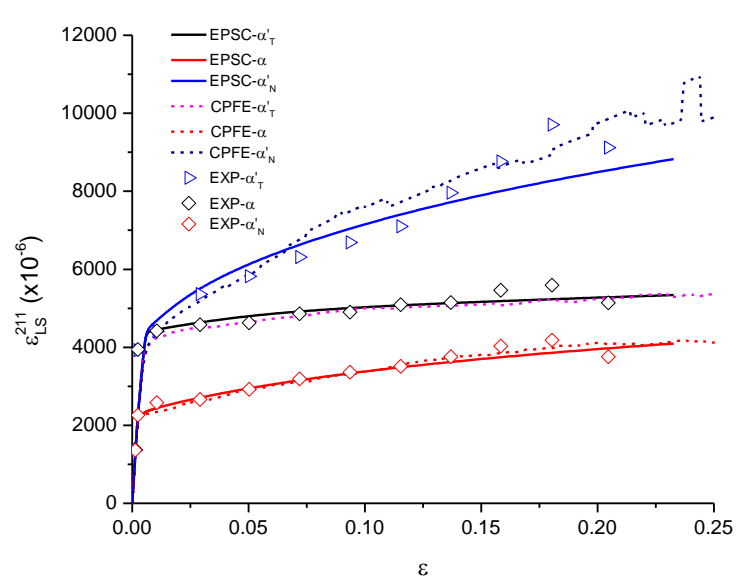

(b)

Fig. 9. HEXRD measured (discrete symbols denoted as EXP), calculated lattice strains from EPSC (solid lines) and CPFE (dashed lines) calculated lattice strains for the three ferrite-like phases with grains whose (a) (200), (b) (211) plane normals are parallel to the LD. The lattice strains obtained from HEXRD and calculated from CPFE are showing more fluctuation relative to EPSC results and this will be discussed later.

In general, the lattice strains for the (200) lattice planes are about 1.7 times those of (211) lattice planes, indicating more lattice stretching along the LD for those martensitic grains with (200) planes perpendicular to the LD. Among the three ferrite-like phases, the lattice strain magnitude trends as follows: new martensite $\left(\alpha_{N}^{\prime}\right)$ $>$ tempered martensite $\left(\alpha_{T}^{\prime}\right)>$ ferrite $(\alpha)$. This indicates that $\alpha_{N}^{\prime}$ has the highest flow stresses, followed by $\alpha_{T}^{\prime}$. Ferrite is the softest phase among the three ferrite-like phases.

\section{Determining Individual Phase slip system Parameters of the QP980 steel}

\subsection{Elastic-Plastic Self-Consistent (EPSC) Method}

The experimental data detailed in Section 3 is next used to obtain individual phase slip system parameters by the use of $\mathrm{CP}$ models via the aforementioned trial-and-error approach. The texture and phase volume fractions obtained from HEXRD will be used to generate inputs for the models, while the comparison between HEXRD measured and predicted lattice strains serve as the slip system parameter selection criterion. The lattice strain for a lattice plane of a specific phase obtained in the in-situ HEXRD tensile tests is an average value for all the crystallites of the phase with their lattice planes $(h k l)$ perpendicular to the diffraction vector ( $S_{h k l}$ in Fig 2.), which is also the direction of strain $\varepsilon_{h k l}$. The lattice strain and stress tensors can be related through the diffraction elastic constants [34] based on various assumptions such as iso-strain (i.e., Voigt) or iso-stress (i.e., Reuss) models. It has been shown previously that neither the Reuss nor the Voigt model can lead to satisfactory individual phase stress-strain relationships in comparison to experimental data $[16,35]$. These methods, therefore, will not be pursued in this study. The EPSC crystal plasticity models [36-38] for tension will be used here initially. With HEXRD measured lattice strains as the parameter selection criterion, the EPSC model has been used as the primary crystal plasticity tool in the literature [36-37] for determining the slip system parameters for individual phases in $1^{\text {st }}$ generation AHSS.

Unlike the Taylor model [33] for a polycrystalline material, which assumes uniform deformation in all grains, a self-consistent scheme, such as EPSC, accounts for the deformation heterogeneity of grains with different crystal 
orientations and shapes, which is considered by solving the Eshelby ellipsoidal inclusion problem [39]. This is accomplished with the approach developed by Hutchinson [36,37] which consists of an extension of the Eshelby tensor of an ellipsoidal inclusion to macroscopically anisotropic materials. The EPSC model starts with an aggregate of grains of different phases of different volume fractions. Those grains have crystal orientations representing that of the texture of each individual phase. Each grain $(\mathrm{g})$ has an instantaneous modulus $\mathbf{M}_{g}$ in the homogeneous equivalent medium with overall moduli M. Each grain undergoes elastic-plastic deformation, and plasticity occurs by dislocation glide of slip systems present in that grain. The behavior of the medium represents that of the whole aggregate of grains. In the model, the interaction equation that links the microscopic strain rate tensor at the grain scale, $\dot{\boldsymbol{\varepsilon}}_{g}$, to the macroscopic strain rate tensor at the polycrystal scale, $\overline{\dot{\boldsymbol{\varepsilon}}}$, is derived in an iterative way via

$\dot{\boldsymbol{\varepsilon}}_{g}=\mathbf{A}_{g} \overline{\dot{\varepsilon}}$

with

$\mathbf{A}_{g}=\left(\mathbf{M}^{*}+\mathbf{M}_{g}\right)^{-1}\left(\mathbf{M}^{*}+\mathbf{M}\right)$

where $\mathbf{M}^{*}$ is introduced as a 'constraint' tensor for a matrix containing an ellipsoidal inclusion with the same orientation and shape as the grain. Here, the generalized Voce law is used to describe the hardening of the $i$-th slip system for each grain:

$\tau_{c}^{i}(\Gamma)=\tau_{0}^{i}+\left(\tau_{1}^{i}+\theta_{1}^{i} \cdot \Gamma\right)\left[1-\exp \left(-\frac{\theta_{0}^{i} \cdot \Gamma}{\tau_{1}^{i}}\right)\right]$

where $\Gamma$ is the total accumulated shear for all systems over the deformation history and $\tau_{c}^{i}$ is the instantaneous critical resolved shear stress (CRSS) as a function of $\Gamma . \tau_{0}^{i}, \tau_{1}^{i}$ and $\theta_{0}^{i}$ are parameters defining the traditional Voce law with $\tau_{0}^{i}$ representing the initial yield CRSS. $\tau_{1}^{i}$ is the difference between saturation and initial yield, and $\theta_{0}^{i}$ is the initial hardening rate. The modification of Voce law is by the addition of the linear hardening term $\theta_{1}^{i} \cdot \Gamma$ with $\theta_{1}^{i}$ representing linear hardening rate which will dominate at large $\Gamma$. The rate of instantaneous $\operatorname{CRSS}, \dot{\tau}_{c}^{i}$, is assumed to be related to the shear rate of the $j$-th slip system, $\dot{\gamma}^{j}$, through

$\dot{\tau}_{c}^{i}=\frac{d \tau_{c}^{i}}{d \Gamma} \sum_{j} h^{i j} \dot{\gamma}^{j}$

where $h^{i j}$ are the hardening coefficients that characterizes "self" (when $i=j$ ) and "latent" (when $i \neq j$ ) hardening between slip systems $i$ and $j$. Since "self" and "latent" hardening could not be distinguished in the diffraction data, a value of 1.0 is used for all components of $h^{i j}$ of all phases considered here.

An incremental stress boundary condition is applied for uniaxial tensile deformation. For each increment, the stress $\left(\boldsymbol{\sigma}^{g}\right)$ and strain tensors $\left(\boldsymbol{\varepsilon}^{g}\right)$ for each grain and the average values for all the grains are calculated $(\overline{\boldsymbol{\sigma}}, \overline{\boldsymbol{\varepsilon}})$. The elastic part of the strain tensors $\left(\boldsymbol{\varepsilon}_{e}^{g}\right)$ for an individual grain is then used to calculate the lattice strain for a plane $(h k l)$ of that grain. Only those grains with their lattice plane normals almost parallel to the loading direction are considered and the average values for the $(h k l)$ plane are used to compare with HEXRD results. An iterative trialand-error approach is then used to identify the slip system parameter set of $\tau_{0}^{i}, \tau_{1}^{i}, \theta_{0}^{i}$ and $\theta_{1}^{i}$ for each phase that 
yields the best match between the EPSC calculated lattice strain with that from in-situ HEXRD detailed in section 3.2 .

\subsection{Crystal plasticity finite element (CPFE) modeling}

Note that the EPSC model does not consider crystal re-orientation during deformation. Furthermore, the selfconsistent scheme only considers the interaction between an individual grain and a medium with average properties of all grains considered. Individual grain-grain or phase-phase interactions are not included. The accuracy of the EPSC-determined individual phase slip system parameters is tested against a CPFE virtual polycrystalline tensile model. A $2^{\text {nd }}$-step CPFE-based parameter refinement is performed with the initial EPSC-estimated parameter set as a reference, a new set of slip system parameters is obtained, expected to be more accurate since CPFE accounts for grain/grain and phase/phase interactions. The CPFE model explicitly resolves individual phases and grains with finite element meshes, and can hence better represent the material heterogeneity on the microstructure scale from the neighboring grain/grain and phase/phase interactions. In addition, grain re-orientation during deformation can also be considered. In this study, a two-step approach is proposed where we use CPFE to refine the initial EPSCdetermined phase slip system parameters.

The CPFE model is implemented in the commercial finite element package ABAQUS V6.10 with a user material subroutine (UMAT) based on the visco-plastic formulation of Asaro et al. [40]. The UMAT is based on the revised version [17, 41] of Huang's code [42]. Revisions to this code enable it to address multiple phases and calculate average lattice strains of crystal planes in each phase in the material. The detailed formulation of this CPFE-UMAT code can be found in the literature [40, 42, 43]. Here, only a brief overview will be given for completeness. In the ABAQUS-UMAT interface, the strain increment and increment of the rigid body rotation are provided at each integration point for each time increment, from which the current strain rate tensor $\mathbf{D}$ and rigid body spin tensor, $\boldsymbol{\Omega}$ can be easily obtained, and hence the current velocity gradient $\mathbf{L}$,

$\mathbf{L}=\mathbf{D}+\mathbf{\Omega}=\mathbf{L}^{e}+\mathbf{L}^{p}$

Note that $\mathbf{L}^{e}$ and $\mathbf{L}^{p}$ are the elastic and plastic parts of the velocity gradient $\mathbf{L}$, respectively, and the plastic part, $\mathbf{L}^{p}$ is related to the slip rates of all the slip systems via

$\mathbf{L}^{p}=\mathbf{D}^{p}+\Omega^{p}=\sum \mathbf{b}^{i} \otimes \mathbf{n}^{i} \dot{\gamma}^{i}$

where $\dot{\gamma}^{i}$ is the shear strain rate of slip system i, and $\mathbf{b}^{i}$ and $\mathbf{n}^{i}$ are the burger's vector and slip plane normal, respectively. $\mathbf{D}^{p}$ and $\boldsymbol{\Omega}^{p}$ are symmetric and antisymmetric part of the plastic velocity gradient tensor, representing plastic part of the strain rate tensor and plastic spin, respectively.

The strain rate tensor can be decomposed into elastic $(e)$ and plastic parts $(p)$, while the rate of spin tensor can be decomposed into lattice $(L)$ and plastic spins $(p)$.

$$
\mathbf{D}=\mathbf{D}^{e}+\mathbf{D}^{p}, \boldsymbol{\Omega}=\boldsymbol{\Omega}^{L}+\boldsymbol{\Omega}^{p}
$$

In the crystal plasticity formulation, the slip rates of various slip systems, $\dot{\gamma}^{i}$ must be determined.

In a visco-plastic crystal plasticity model, the shear strain rate on each system $i$ is assumed to be related to the resolved shear stress $\tau^{i}$ and the CRSS, $\tau_{c}^{i}$ (also referred to as the slip resistance), by: 
$\dot{\gamma}^{i}=\dot{\gamma}_{0}\left|\frac{\tau^{i}}{\tau_{c}^{i}}\right|^{\frac{1}{m}} \operatorname{sign}\left(\tau^{i}\right)$

where $\dot{\gamma}_{0}$ is the reference shear strain rate (a constant), and $m$ is a rate sensitivity coefficient. Similar to the EPSC model, the CRSS, $\tau_{c}^{i}$, at each integration point of the finite element model are calculated by the integration of Eqn. (7) where the modified Voce law (Eqn. (6)) is used for hardening. As long as the slip rates are obtained, the plasticity part of velocity gradient $\left(\mathbf{L}^{p}\right)$ can be found from equation (9) as well as the plastic parts of the deformation gradient $\left(\mathbf{D}^{p}\right)$ and spin tensor $\left(\boldsymbol{\Omega}^{p}\right)$. The rate of elastic deformation $\left(\mathbf{D}^{e}\right)$ and lattice spin $\left(\boldsymbol{\Omega}^{L}\right)$ can be calculated from equations (10), from which the total elastic strain tensor $\left(\boldsymbol{\varepsilon}^{e}\right)$ and crystal orientation for each integration point can be updated at the end of each time increment. The new stress tensor can be calculated from the stress tensor at the start of the time increment provided from the Abaqus-UMAT interface and $\mathbf{D}^{e}$ based on Hooke's law [42]. Since there is no temperature change considered, the lattice distortion is a result only of elastic deformation from which the lattice strains can be calculated.

\section{Results and Discussion}

\subsection{EPSC modeling}

An aggregate of 40,000 grains is considered as the representative volume element for the QP980 material which consists of four phases: $\alpha, \alpha_{\mathrm{T}}^{\prime}, \gamma$, and $\alpha_{\mathrm{N}}^{\prime}$. The total volume fraction of austenite and $\alpha_{\mathrm{N}}{ }_{\mathrm{N}}$ is assumed to be $11.5 \%$ (4660 grains), as suggested from the results in Fig. 5. Based on the calculated volume fractions of the initial ferrite-like phases, there will be 14720 ferrite grains and 20680 tempered martensite grains.

As stated previously, the $\alpha^{\prime}$ is assumed to exist at the outset of deformation with a volume fraction of 5.75\% (2300 grains) and another $5.75 \%$ (2300 grains) is assumed to be austenite in the model. All 37700 grains of the three ferrite-like phases $\left(\alpha+\alpha_{T}^{\prime}+\alpha_{N}^{\prime}\right)$ are generated based on the texture of the ferrite-like phases (see Fig. 6(a)) calculated from HEXRD measurement through the MTM-FHM texture analysis software package [31]. Similarly, the 2300 austenite grains are generated based on the texture of austenite (see Fig. 6(b)). The slip systems that contribute to deformation of the ferrite-like phases are assumed to be $(110)<111\rangle,(112)<111\rangle$ and $(123)<111\rangle$, while the slip systems for the austenite are $(111)<110\rangle$. With the EPSC model described above and the trial and error method described in Section 4.1, a set of slip system parameters for the ferrite-like phases and austenite phase are obtained and shown in Table 2.

Table 2. The slip system parameters $\left(\tau_{0}, \tau_{1}, \theta_{0}, \theta_{1}\right)$ as in Eqn. (6)) all in MPa, obtained from EPSC modeling with an iterative trial-and-error approach in which the EPSC calculated lattice strains are compared with HEXRD measured lattice strains during uniaxial tension (Figs. 7(b) and 9).

\begin{tabular}{|l|l|l|l|l|}
\hline Phase & $\tau_{0}$ & $\tau_{1}$ & $\theta_{0}$ & $\theta_{1}$ \\
\hline$\alpha$ & 200 & 150 & 500 & 80 \\
\hline$\alpha^{\prime}{ }_{T}$ & 430 & 50 & 500 & 50 \\
\hline$\alpha^{\prime}{ }_{N}$ & 440 & 40 & 2200 & 2200 \\
\hline$\gamma$ & 240 & 1 & 800 & 450 \\
\hline
\end{tabular}


Using these parameters, the EPSC calculated lattice strains (solid lines in Fig. 9(b)) for the ferrite-like phase grains with their (211) lattice plane normals parallel to the LD correspond well with those obtained from HEXRD data (discrete symbols in Fig. 9(b)). In general, it is also true for grains with their (200) lattice plane normals parallel to the LD, see Fig. 9(a). Among the martensite phases, the calculated lattice strains of the $\alpha^{\prime}{ }_{N}$ show more deviation from the HEXRD results relative to the $\alpha^{\prime}$. This can be attributed to the fact that austenite to martensite phase transformation is not considered in the EPSC model. Fig. 9(c) shows measured and calculated lattice strains for the austenite grains whose (200) or (220) lattice plane normals parallel to the LD. Although the large differences between the (200) and (220) lattice strains are captured by the EPSC model results in general (solid lines in Fig. 10), the evolution of lattice strain calculated for the (220) lattice strains (red solid line in Fig. 9(c)) show a big difference with those from the HEXRD experiment.

\subsection{Using EPSC-estimated Slip System Parameters in CPFE models.}

In this section, we will use the EPSC-estimated phase slip system parameters in Table 2 as input to a CPFE model. The macroscopic stresses and lattice strains versus macroscopic strains will be calculated and the results will be compared with those results obtained from EPSC calculations and HEXRD measurements. Both CPFE and EPSC use this same set of parameters as shown in Table 2. The average phase stresses vs average phase strains from CPFE and EPSC will also be compared since no direct experimental results are available.

Fig. 10(a) shows reasonably good agreement between the EPSC-predicted macro tensile properties (solid line) with parameters in Table 2 and the experimentally measured stress-strain curve (discrete symbol). In both EPSC and CPFE, the macro stress-strain curve during defomation is calculated by averaging the $\sigma_{11}$ and $\varepsilon_{11}$ over all the grains via,

$$
\sigma=\bar{\sigma}_{11}=\sum_{i=1}^{N} \sigma_{11}^{i}, \quad \varepsilon=\bar{\varepsilon}_{11}=\sum_{i=1}^{N} \varepsilon_{11}^{i}
$$

where $\sigma_{11}$ is the tensile flow stress along the LD. Here $\mathrm{N}=40,000$ represents the total number of grains in the EPSCconsidered grain aggregate.

The average stress versus strain curve for phase $j\left(\sigma^{j}\right.$ vs $\left.\varepsilon^{j}\right)$ are calculated similarly, but only averaring over all the grains belonging to that particular phase:

$$
\sigma^{j}=\bar{\sigma}_{11}^{i, j}=\sum_{i=1}^{N_{j}} \sigma_{11}^{i, j}, \quad \varepsilon^{j}=\bar{\varepsilon}_{11}^{i, j}=\sum_{i=1}^{N_{j}} \varepsilon_{11}^{i, j}
$$

Here $N_{j}$ is the number of grains belonging to phase $j\left(=\alpha, \alpha_{\mathrm{T}}^{\prime}, \alpha_{\mathrm{N}}^{\prime}\right.$ and $\left.\gamma\right)$. The average phase stress-phase strain curves obtained from the EPSC model are shown in Fig. 10(b) as the heavier symbols/lines, with different colors representing the $\alpha, \alpha_{\mathrm{T}}^{\prime}, \alpha^{\prime}{ }_{\mathrm{N}}$ and $\gamma$ phases. 


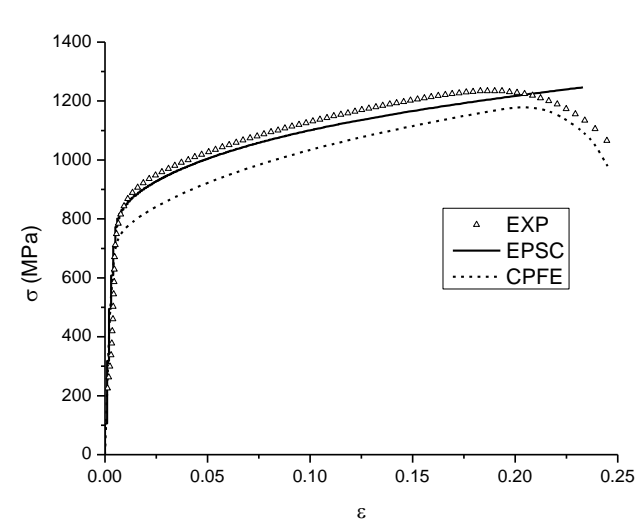

(a)

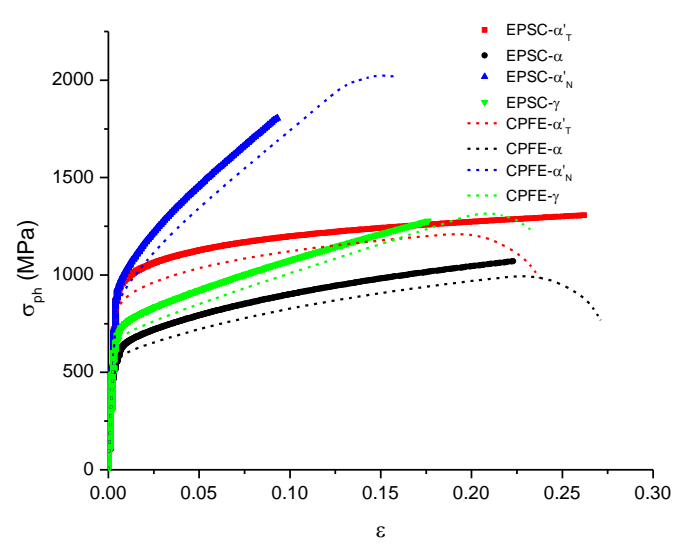

(b)

Fig. 10. (a) The macro (average) true stress- strain curves for QP980 and (b) phases stress-strain curves for the phases calculated by the EPSC and CPFE models using the slip system parameters in Table 2. The macro curves are compared with experimental (EXP) results.

The CPFE-predicted macro stress vs. strain and individual phase stress vs. strain curves are also shown in Figs. 10 as dashed lines. The ABAQUS CPFE model, which is shown in Fig.11 (a) as a tensile sample, contains a rectangular domain with $10 \times 30 \times 90$ 8-noded brick elements with reduced integration $(\mathrm{C} 3 \mathrm{D} 8 \mathrm{R})$ with each element representing a grain with a specific crystal orientation. The HEXRD measured texture for austenite and ferrite-like phases is used to generate grain orientations which are assigned to each grain (or element). Ideally, realistic grain structure representation of experimentally derived microstructure in finite element models are desirable for more accurate calculations. Such a model, however, would be computationally prohibitive if sufficient numbers of grains are to be considered. Displacement-controlled tensile loading is simulated by imposing displacement boundary conditions on the left and right free surfaces of the sample in Fig. 11(a) along the loading direction (LD). It needs to be noted that no constraints along both the transverse (TD) and normal (TD) directions are applied to both surfaces; therefore the model used here can be deemed as a unit cell model representing any part of a tensile specimen in the gauge section and the location of necking can appear anywhere along LD. The results in Fig. 10(a) show that the CPFE-calculated macroscopic stress-strain curve is about 8\% lower than that obtained from EPSC and $10.5 \%$ lower than the experimental results from the in situ HEXRD test. In addition to the initial yield and the hardening behaviors of the polycrystal, the CPFE model can also predict the peak stress (Fig. 10(a)) and necking in the virtual sample (see Fig. 11(b)) as a result of deformation instability. Fig. 10(b) shows that similar disprepancies are observed between the EPSC (dashed) and the CPFE (heavier symbols/lines) predicted average phase stress-strain curves along the LD. Overall, the CPFE-predicted flow stress is about 9-10\% and 8\% lower than the EPSCpredicted value for the ferrite-like phases and the austenite phase, respectively. 


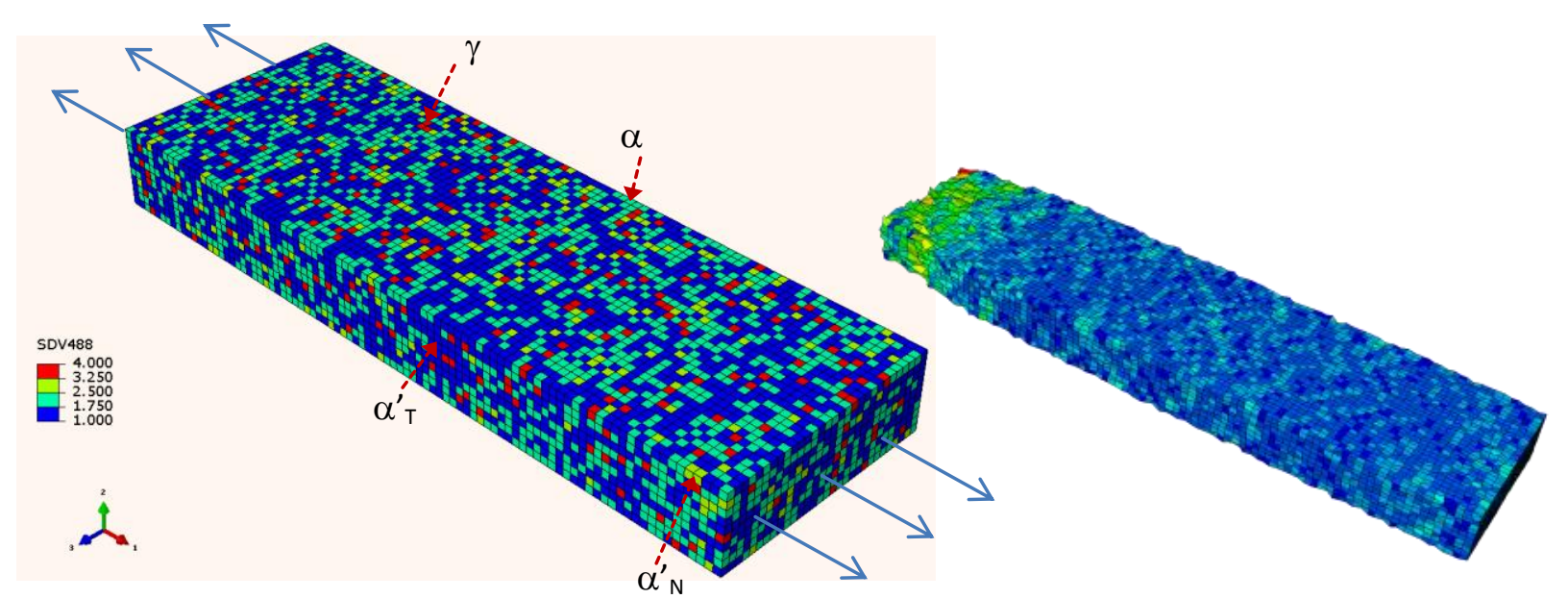

(a)

(b)

Fig. 11. The crystal finite element (CPFE) QP980 tension model which shows: (a) the 4 individual phases (Blue: $\alpha_{T}^{\prime}$ Light blue: $\alpha$, yellow: $\alpha^{\prime}$ and red: $\gamma$ ), and (b) the equivalent strain contour after necking.

In addition, the lattice strains calculated from the CPFE model are also lower than those from the EPSC model (dashed lines in Figs. 9). This is most obvious for the (200) lattice strains for all the phases, while the lattice strains for the (211) for the ferrite-like phases and (220) for the austenite are much closer between the two models. Another observation is that more fluctuations (zig-zags, to be discussed shortly) in lattice strains arise from the CPFE model, while the EPSC-predicted lattice strains monotonically increase with deformation. The HEXRD measured lattice strains also show some degree of fluctuations, see Fig. 9.

While the EPSC model does not consider grain orientation changes during plastic deformation, the CPFE model, however, updates grain orientation and therefore can calculate texture changes of the various phases during deformation. Figs. 12(a)-(c) show the pole figures for the ferrite-like phases $\left(\alpha, \alpha_{\mathrm{T}}^{\prime}, \alpha_{\mathrm{N}}^{\prime}\right)$, and Fig. 12(d) shows HEXRD diffraction rings at 0.15 true tensile strain. The results show that a (110) fibre texture is developed for the ferrite-like phases (Fig. 12(a)-(c)). The calculated texture evolutions are typical for BCC (BCT). The pole figure for the austenite is not plotted here, since most of them have already transformed in actual tenile deformation. The differences between the deformation textures of the three ferrite-like like phases are not significant. Compared with the initial texture (Fig. 6(a)), the intensity at the LD pole is much higher for the ferrite-like phases, indicating more grains rotated to have 110 lattice plane normals parallel to the loading direction. This is consistent with the Debye ring intensity of (110) planes become much stronger along the LD direction (Compare Figs. 3(a) and 12(d)).

As discussed previously and shown in Fig. 10, the CPFE calculated individual phase stress and the overall stress levels are consistently lower than those calculated from the EPSC model with the same slip system parameters in Table 2. The reason for this discrepancy can be attributed to the secant approach in the adopted EPSC formulation. Several formulations exist in the self-consistent (SC) method that relates stress and strain rates in each grain with the average values of the effective medium, including the stiff secant approach [36], the compliant tangent approach [44] and the intermediate approximation [45], where the last approach yields the response of a cluster of polycrystals that lies in-between the secant and the tangent approaches [46]. Since the EPSC model adopted in the current study is based on Hutchinson's formulation with the secant approach, it yields a stiffer solution, i.e. higher value of stresses (and hence lattice strains) than other formulations. 


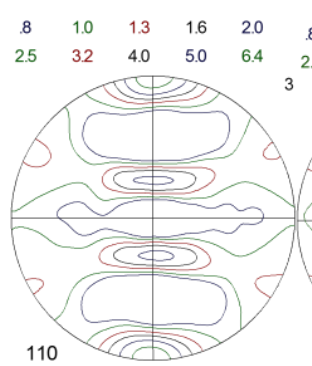

(a) $110 \alpha_{T}^{\prime}$

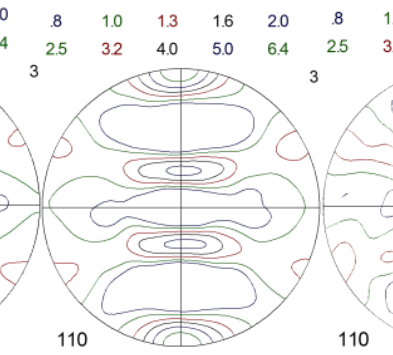

(b) $110 \alpha$

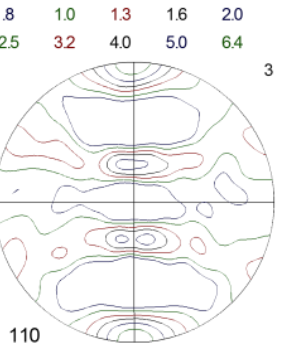

(c) $110 \alpha^{\prime}$
110 Diffraction ring

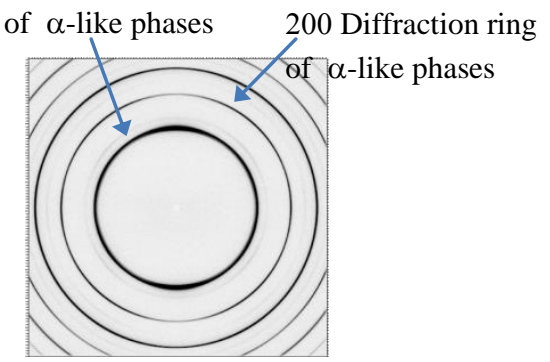

(d) HEXRD diffraction Ring

Fig. 12. (a)-(c) The (110) pole figures for the ferrite-like phases $\left(\alpha_{T}^{\prime}, \alpha\right.$ and $\left.\alpha_{N}^{\prime}\right)$ and (d) the HEXRD diffraction rings at a tensile strain of 0.15 calculated from the CPFE model, the scale at top of pole figures of the pole figures are intensity contour levels.

\subsection{Refinement of Slip System Parameters}

Because the CPFE model can explicitly consider the grain/grain and phase/phase interactions as well as the texture evolution during deformation (EPSC does not), we expect that it will provide more accurate predictions of the macro-scale flow behavior of the QP980 steel once the individual phase slip system parameters are accurately determined. However, the discrepancies between the CPFE-predicted stress vs. strain curve and experimental measurement in Fig. 10 indicate that the slip system parameters listed in Table 2 are slightly lower than the actual values for the various phases in QP980. A further refinement of the parameters therefore is needed.

Through trial-and-error, we found that when the $\tau_{0}$ values listed in Table 2 are increased to 466, 218 and 271 MPa for the $\alpha_{\mathrm{T}}^{\prime}, \alpha$, and $\gamma$ phases, respectively and that for the $\alpha_{\mathrm{N}}^{\prime}$ phase is kept unchanged, the CPFE-calculated macro stress-strain curve is in excellent agreement with the EPSC and HEXRD experimental results, see Fig. 14(a). Furthermore, it seems that the location for the peak stress in Fig. 14(a) from CPFE is very close to that of the HEXRD measurements at $\sim 19 \%$ strain. The refined slip system parameter set is listed in Table 3 . The average phase stress-strain curves (Fig. 14(b)) for the various phases are very close between the CPFE model with parameters in Table 3 and those calculated from the EPSC model using the parameters in Table 2.

The CPFE-calculated (200), (211) lattice strains for the ferrite-like phases in Fig. 15 (a) and (b) respectively, and the (200), (220) lattice strains for the austenite in Fig. 15 (c) are also closer to those of the HEXRD experiments. The trend in the variation of the (220) austenite lattice strains (the red dashed line in Fig. 15(c)) is much closer to that of the HERXD experiments (the red triangular dots in Fig. 15(c)) than the EPSC model (the red solid line in Fig. $15(\mathrm{c}))$. 


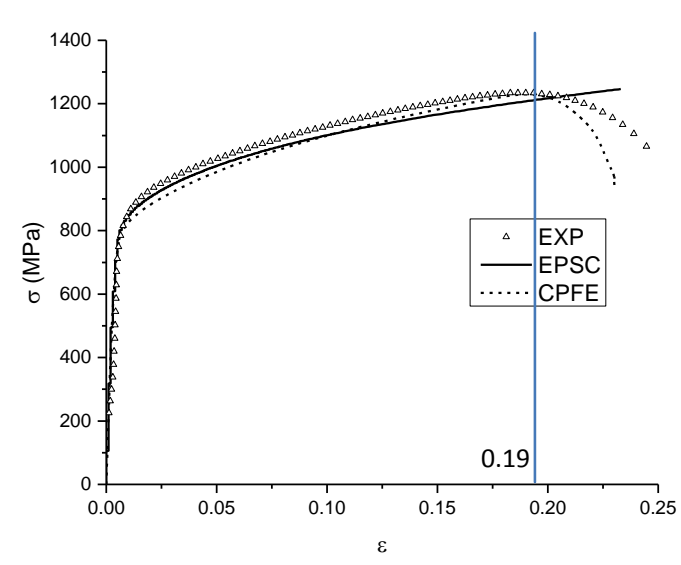

(a)

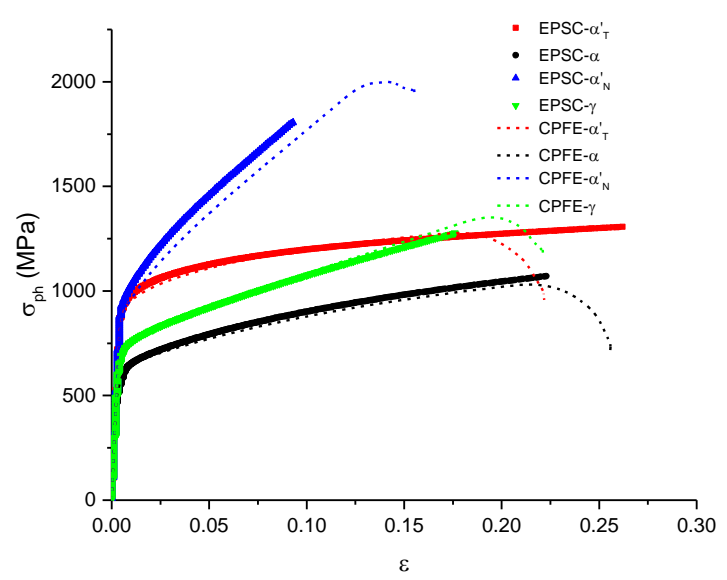

(b)

Fig. 14. (a) The macro stress-strain curves (b) average phase stress-strain curves calculated by EPSC using parameters in Table 2 and CPFE using parameters in Table 3, respectively. The model calculated macro stress-strain curves are also compared with the experimental curve from HEXRD in (a).

Table 3. The refined slip system parameters, $\tau_{0}, \tau_{1}, \theta_{0}, \theta_{1}$, via CPFE modeling, all in MPa.

\begin{tabular}{|l|l|l|l|l|}
\hline Phase & $\tau_{0}$ & $\tau_{1}$ & $\theta_{0}$ & $\theta_{1}$ \\
\hline$\alpha$ & 218 & 150 & 500 & 80 \\
\hline$\alpha_{\mathrm{T}}^{\prime}$ & 466 & 50 & 500 & 50 \\
\hline$\alpha_{\mathrm{n}}^{\prime}$ & 440 & 40 & 2200 & 2200 \\
\hline$\gamma$ & 271 & 1 & 800 & 450 \\
\hline
\end{tabular}

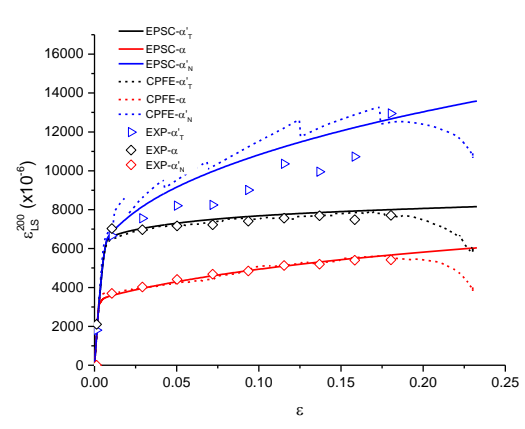

(a)

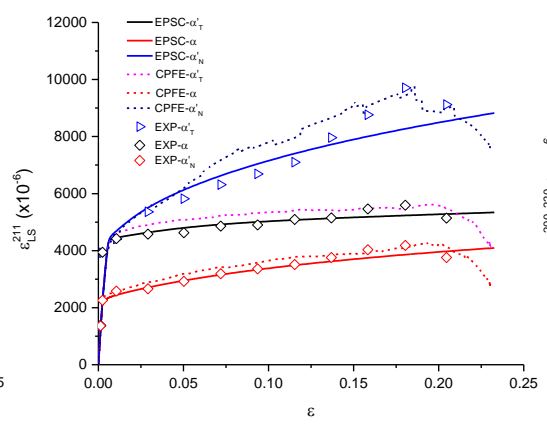

(b)

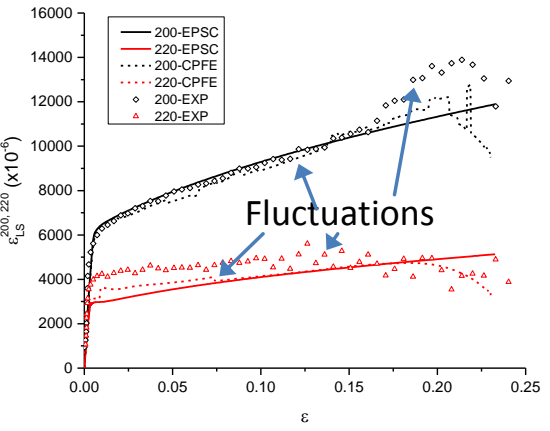

(c)

Fig. 15. The CPFE-calculated lattice strains vs. macro-strain with $\tau_{0}$ in Table 3 for: (a) (200) planes and (b) (211) planes of the $\alpha$ like phases, (c) both (200) and (220) planes of $\gamma$, in comparison with EPSC model results using $\tau_{0}$ in Table 2 and HEXRD experimental data.

Another interesting observation from the results in Fig. 15 is the CPFE-calculated fluctuations of lattice strain with respect to macro-strain (dashed lines), whereas the EPSC-predictions show smooth and monotonic lattice strain increase as deformation progresses (solid lines). Such lattice strain fluctuations are observed in the HEXRD measured lattice strains (discrete symbols). The fluctuation in the CPFE predictions can be traced to considerations of grain re-orientation in the model. Since no grain re-orientation is considered in the EPSC model, those grains with a specific lattice plane normals parallel to loading direction (within a certain tolerance) at the start of calculation are always included in the calculation of lattice strains. Let us denote those grains in a phase with the specific $h k l$ plane normal falling within $2^{\circ}$ of the loading direction, i.e. $\beta=88 \sim 92^{\circ}$, as GT (Grains within angular Tolerance of $\pm 2^{\circ}$ ) and 
the rest of the grains as GNT (Grains not within an angular Tolerance of $\pm 2^{\circ}$ ). During deformation, grains rotate and re-orient themselves due to rigid body and plastic spins, and when this is considered as in the CPFE model, some GT grains can become GNT and vice versa. Fig. 16 shows the normalized number of GT grains of various lattice planes, $N_{G T}^{h k l}(h k l=110,200$ or 211), in the ferrite-like phases as a function of macro-strain. The normalized number of GT grains is the number of GT grains during deformation divided by the initial number of GT grains before deformation for the various lattice planes. $N_{G T}^{110}$ (the heavy dotted lines in Fig. 16) of all three ferrite-like phases increase with deformation, corresponding to the formation of a 110 fibre texture calculated by the CPFE model as shown in Fig. 12(a)-(c). It is also reflected on the 110 Debye ring in the HEXRD results (Fig. 12(d)) which show more 110 intensity at the location corresponding to the loading direction. $N_{G T}^{200}$ grains of ferrite appear to experience the most dramatic changes relative to other ferrite grains. It initially increases rapidly and then decreases to a level close to its initial value. A large variation is also found for the number of 200 GT grains for the new martensite phase, consistent with the several sudden drops of 200 lattice strains calculated by the CPFE model as shown in Fig. 12 (a). The number of 200 GT grains for $\alpha_{T}^{\prime}$ shows the same trend as $\alpha$, but changes more gradually and its final value is about of the initial one. $N_{G T}^{211}$ grains, on the other land, decrease gradually with deformation. The above discussions indicate that the lattice strain fluctuation observed from HEXRD is most probably due to the crystal reorientation during deformation.

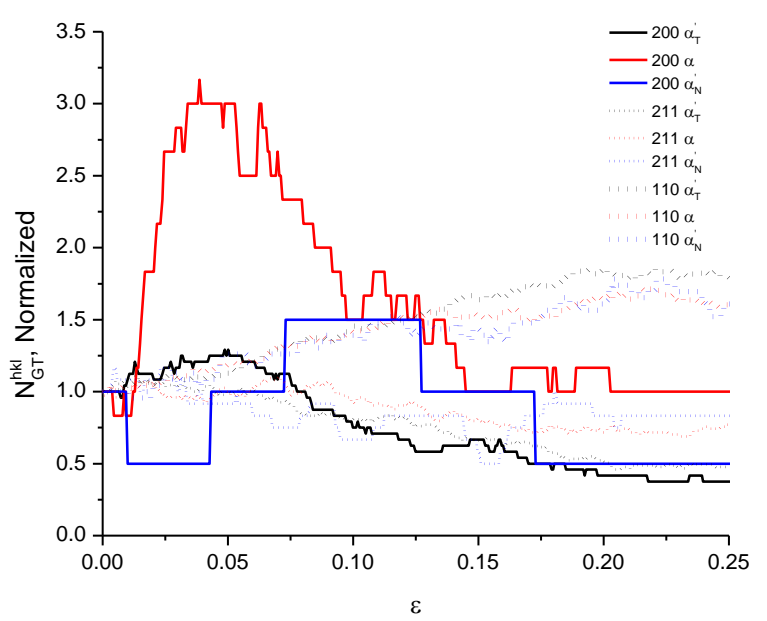

Fig. 16. (a) Normalized number of GT grains for various lattice planes vs. the macro-strain

Note that neither the EPSC nor the CPFE model has considered martensitic phase transformation and its influence on the lattice distortion during loading, and this may explain the discrepancies between the experimentally measured lattice strains and the calculated values for $\alpha_{T}^{\prime}$ and $\gamma$. This is especially notable for the 200 martensite and 220 austenite planes, as shown in Fig. 15. In fact, no studies have been reported in the literature wherein lattice strains are computed while considering the martensitic transformation directly in CPFE models. Further development is needed to directly model the martensitic transformation in the crystal plasticity formulations in order to obtain more accurate individual phase properties in multiphase TRIP-assisted steels.

It must be noted that we have only used the lattice strains of HEXRD measurement along the loading direction (LD) for the determination for slip system parameters for the individual phases in the current work. From 
the HEXRD measurement, the lattice strains for all directions on the plane normal to the incident beam can be obtained. Other than the loading direction (LD), the transverse direction (TD) is another important direction. The elastic strains along the TD are usually compressive and smaller than those along the LD based on the Poisson's ratio. The smaller strains along the TD make deconvolution of the overlapping peaks between the ferrite-like phases more difficult. Therefore, the lattice strains along the TD are not used in the process of obtaining the slip system parameters. Here the HEXRD measured peaks for the ferrite-likes can only be separated into two peaks during peak fitting and the resultant lattice strains for (200) and (211) obtained this way are shown in Fig. 17(a) and (b) in open symbols, while for comparison, the CPFE model calculated results for the three ferrite-like phases are denoted by the dashed lines. The lattice strains of HEXRD results shown by the black triangular open symbols are small and are close to the results of ferrite calculated from the CPFE models (the black dashed lines) for both the 200 and 211 planes. The lattice strains of HEXRD results shown by the red square open symbols are in between those CPFE calculated values of the tempered martensite $\left(\alpha_{\mathrm{T}}^{\prime}\right)$ and new martensite $\left(\alpha_{\mathrm{N}}^{\prime}\right)$.

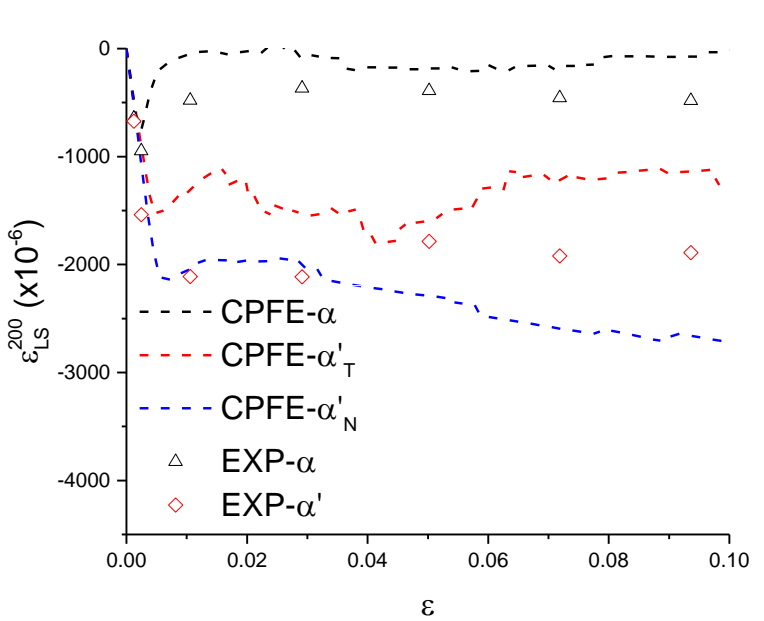

(a)

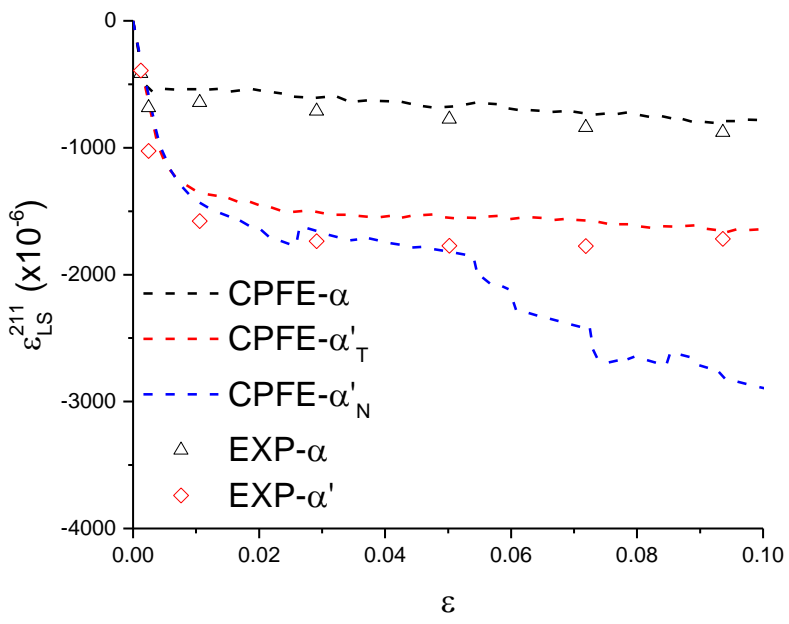

(b)

Fig. 17. The CPFE-calculated lattice strains along TD vs. macro-strain for: (a) (200) planes and (b) (211) planes of the $\alpha$-like phases in comparison with and HEXRD experimental data.

As for the austenite (Fig. 18), the CPFE calculated lattice strains for the (220) planes correlate very well with results obtained from HEXRD measurement, while those for the (200) planes do not correlate well between the CPFE calculations and HEXRD measurements. A contributing factor is the absence of the austenite-martensite phase transformation in the models. 


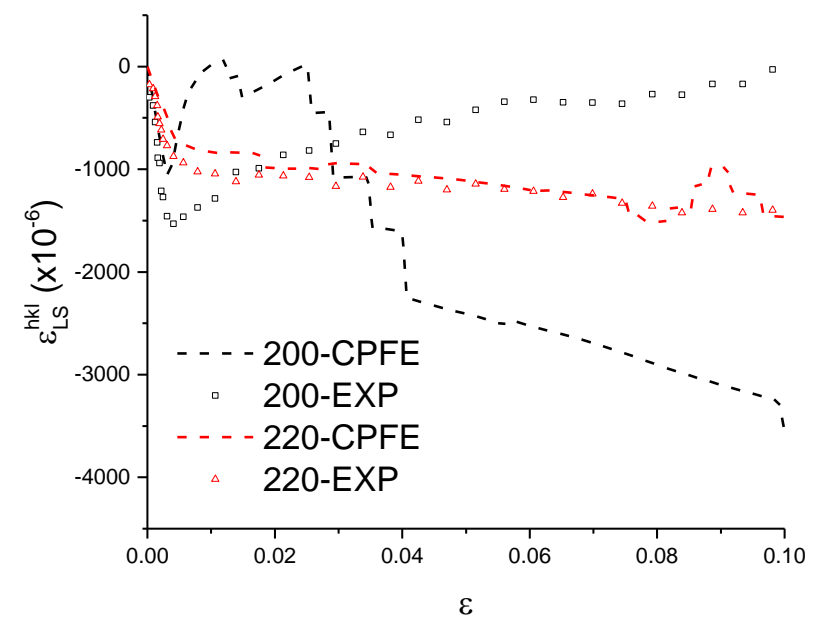

Fig. 18. The CPFE-calculated lattice strains along TD vs. macro-strain for both (200) and (220) planes of $\gamma$, in comparison with and HEXRD experimental data.

\section{Summary Remarks}

In this study, in-situ HEXRD has been utilized to measure the austenite transformation kinetics, lattice strains and phase volume fractions and initial textures during tensile deformation of a multiphase TRIP-assisted steel. Both EPSC and CPFE models are utilized to determine slip system parameters for the constituent phases in the QP980, viz. the $\alpha$-like phases $\left(\alpha, \alpha_{T}^{\prime}, \alpha_{N}^{\prime}\right)$ and retained austenite $(\gamma)$. The results for both models are critically compared with experimental results. The following conclusions can be drawn:

1. With the EPSC-estimated slip system parameters as inputs (see Table 2), polycrystal CPFE model will yield lower macro stresses and phase lattice strains in respect to macro-strains than the experimental measurements.

2. The slip system parameters are refined based on a second CPFE-based modeling step. With the refined parameters (see Table 3) for the various phases in the alloy, the polycrystal CPFE model yields good predictions in terms of macroscopic stress-strain curves and lattice distortion compared with experimental results.

3. The trend of HEXRD obtained lattice strain variation of (220) plane of austenite $(\gamma)$ is much better captured by CPFE than EPSC.

4. The EPSC calculation is more computationally efficient than the CPFE model and it is still good for the initial estimation of the slip system parameters which can be later fine-tuned based on CPFE modeling. The EPSC-estimated average phase stress-strain curves may be used for microstructure-based finite element model where no grain level heterogeneity, i.e. crystal anisotropy, is considered.

No martensitic transformation is considered in the current work due to the difficulty of directly modeling such transformation and its impact on lattice distortions during deformation in crystal plasticity models. No existing work has been reported in the literature for such an effort. In this work, the retained austenite is assumed to have already partly transformed to new martensite before the EPSC or CPFE simulations. Since the volume fraction of austenite $\sim 12 \%$ is still relatively small in the current QP980 steel, the results in this study show that such an 
assumption can still yield good results compared with experimentally measured stress vs strain curves and lattice strain curves.

Quantifying the individual phase plastic flow properties is a critical link for the overall performance predictions of multiphase materials in the integrated computational materials engineering (ICME) framework. This study details a coupled experimental (HEXRD) and modeling approach in determining the critical resolved shear stresses and hardening parameters of the constituent phases in a TRIP-assisted steel. Two different models (EPSC and CPFE) are used, and the resulting critical resolved shear stresses and hardening parameters are different due to the different assumptions used. These results indicate that, from the ICME perspective, consistent assumptions must be ensured when passing data from one scale to the next scale.

\section{Acknowledgements}

This material is based upon work supported by the Department of Energy National Energy Technology Laboratory under Award Number No. DE-EE0005976. This report was prepared as an account of work sponsored by an agency of the United States Government. Neither the United States Government nor any agency thereof, nor any of their employees, makes any warranty, express or implied, or assumes any legal liability or responsibility for the accuracy, completeness, or usefulness of any information, apparatus, product, or process disclosed, or represents that its use would not infringe privately owned rights. Reference herein to any specific commercial product, process, or service by trade name, trademark, manufacturer, or otherwise does not necessarily constitute or imply its endorsement, recommendation, or favoring by the United States Government or any agency thereof. The views and opinions of authors expressed herein do not necessarily state or reflect those of the United States Government or any agency thereof. Such support does not constitute an endorsement by the Department of Energy of the work or the views expressed herein. Pacific Northwest National Laboratory is operated by Battelle Memorial Institute for the U.S. Department of Energy under Contract no. DE-AC06-76RL01830. This research used resources of the Advanced Photon Source, a U.S. Department of Energy (DOE) Office of Science User Facility operated for the DOE Office of Science by Argonne National Laboratory under Contract No. DE-AC02-06CH11357.

\section{References}

[1] R. Kuziak, R. Kawalla, S. Waengler, Advanced high strength steels for automotive industry, Archives of Civil and Mechanical Engineering 8 (2008), 103-117.

[2] W. J. Joost, Reducing Vehicle Weight and Improving U.S. Energy Efficiency Using Integrated Computational Materials Engineering, JOM 64 (2012), 1032-1038.

[3] S. C. Baik, S. J. Kim, Y. S. Jin, O. Kwon, Effects of Alloying Elements on Mechanical Properties and Phase Transformation of TRIP Cold Rolled Steel Sheets, SAE Technical Paper 2000-01-2699 (2000).

[4] P. D. Zavattieri, V. Savic, J. Hector, J. R. Fekete, W. Tong, Y. Xuan, Spatio-temporal characteristics of the Portevin-Le Chatelier effect in austenitic steel with twinning induced plasticity, International Journal of Plasticity 25 (2009), 2298-2330.

[5] J. Speer, D. K. Matlock, B. C. De Cooman, J. G. Schroth, Carbon partitioning into austenite after martensite transformation, Acta Materialia 51 (2003), 2611-2622.

[6] A. J. Clarke, J. G. Speer, D. K. Matlock, F. C. Rizzo, D. V. Edmonds, M. J. Santofimia, Influence of carbon partitioning kinetics on final austenite fraction during quenching and partitioning, Scripta Materialia 61 (2009), 149-152.

[7] E. DeMoor, P. J. Gibbs, J. G. Speer, D. K. Matlock, Strategies for Third Generation Advanced High Strength Steel Development, AIST Transactions 7 (2010), 133.

[8] V. Savic, L. Hector, H. Essat, A. Sachdev, J. Quinn, R. Krupitzer, X. Sun, Integrated Computational Materials Engineering (ICME) for Third Generation Advanced High-Strength Steel Development, SAE Technical Paper 2015-01-0459 (2015). 
[9] N. Jia, R. Lin Peng, Y. D. Wang, S. Johansson, P. K. Liaw, Micromechanical behavior and texture evolution of duplex stainless steel studied by neutron diffraction and self-consistent modeling, Acta Materialia 56 (2008), 782-793.

[10] N. Jia, Z. H. Cong, X. Sun, S. Cheng, Z. H. Nie, Y. Ren, P. K. Liaw, Y. D. Wang, An in situ high-energy Xray diffraction study of micromechanical behavior of multiple phases in advanced high-strength steels, Acta Materialia 57 (2009), 3965-3977.

[11] Z. H. Cong, N. Jia, X. Sun, Y. Ren, J. Almer, Y. D. Wang, Stress and Strain Partitioning of Ferrite and Martensite during Deformation, Metall and Mat Trans A 40 (2009), 1383-1387.

[12] M. Lentz, M. Klaus, R. S. Coelho, N. Schaefer, F. Schmack, W. Reimers, B. Clausen, Analysis of the Deformation Behavior of Magnesium-Rare Earth Alloys Mg-2-pct Mn-1-pct Rare Earth and Mg-5-pct Y-4pct Rare Earth by In Situ Energy-Dispersive X-ray Synchrotron Diffraction and Elasto-Plastic SelfConsistent Modeling, Metallurgical and Materials Transactions A 45 (2014), 5721-5735.

[13] B. Clausen, T. Lorentzen, T. Leffers, Self-consistent modelling of the plastic deformation of f.c.c. polycrystals and its implications for diffraction measurements of internal stresses, Acta Materialia 46 (1998), 3087-3098.

[14] B. Clausen, D. W. Brown, M. A. M. Bourke, T. A. Saleh, S. A. Maloy, In situ neutron diffraction and ElasticPlastic Self-Consistent polycrystal modeling of HT-9, Journal of Nuclear Materials 425 (2012), 228-232.

[15] A. A. Saleh, E. V. Pereloma, B. Clausen, D. W. Brown, C. N. Tome, A. A. Gazder, Self-consistent modelling of lattice strains during the in-situ tensile loading of twinning induced plasticity steel, Materials Science and Engineering: A 589 (2014), 66-75.

[16] X. H. Hu, K. S. Choi, X. Sun, Y. Ren, Y. D. Wang, Determining individual phase flow properties in a Quench and Partitioning steel with in-situ high energy X-ray diffraction and multi-phase elasto-plastic selfconsistent method, Metall and Mat Trans A 47 (2016), 5733-5749.

[17] X. H. Hu, D. S. Wilkinson, M. Jain, P. D. Wu, R. K. Mishra, The impact of particle distributions and grainlevel inhomogeneities on post-necking deformation and fracture in AA5754 sheet alloys during uniaxial tension, Materials Science and Engineering: A 528 (2011), 2002-2016.

[18] X. H. Hu, M. Jain, P. D. Wu, D. S. Wilkinson, R. K. Mishra, A macro-micro-multi-level modeling scheme to study the effect of particle distribution on wrap-bendability of AA5754 sheet alloys, Journal of Materials Processing Technology 210 (2010), 1232-1242.

[19] W. Liu, K. Choi, A. Solami, X. Sun, Effects of Forming Induced Phase Transformation on Crushing Behavior of TRIP Steel, SAE Technical Paper 2010-01-0216 (2010).

[20] J. Coryell, V. Savic, L. Hector, S. Mishra, Temperature Effects on the Deformation and Fracture of a Quenched-and-Partitioned Steel, SAE Technical Paper 2013-01-0610 (2013).

[21] X. C. Xiong, B. Chen, M. X. Huang, J. F. Wang, L. Wang, The effect of morphology on the stability of retained austenite in a quenched and partitioned steel, Scripta Materialia 68 (2013), 321-324.

[22] W. H. Bragg, W. L. Bragg, The Reflection of X-rays by Crystals, Proceedings of the Royal Society of London. Series A 88 (1913), 428-438.

[23] J. Coryell, V. Savic, L. Hector, S. Mishra, Temperature Effects on the Deformation and Fracture of a Quenched-and-Partitioned Steel, SAE Technical Paper 2013-01-0610 (2013).

[24] F. Abu-Farha, X. H. Hu, Y. Ren, X. Sun, L. G. Hector, In-situ measurements of retained austenite transformation kinetics via coupled digital image correlation and high energy X-ray diffraction, In Preparation (2015).

[25] A. P. Hammersley, FIT2D V12. 012 Reference Manual V6. 0, ESRF International Report No. ESRF98HA01T. Program available at http://www. esrf. eu/computing/scientific/FIT2D (2004)

[26] P. M. Edwards, Origin 7.0: scientific graphing and data analysis software, Journal of chemical information and computer sciences 42 (2002), 1270-1271.

[27] T. Gnaupel-Herold, A. Creuziger, Diffraction study of the retained austenite content in TRIP steels, Materials Science and Engineering: A 528 (2011), 3594-3600.

[28] ASTM, Standard Practice for X-Ray Determination of Retained Austenite in Steel with Near Random Crystallographic Orientation, ASTM Standards E 975 - 03 (1980).

[29] C. Kim, X-ray method of measuring retained austenite in heat treated white cast irons, J. Heat Treating 1 (1979), 43-51.

[30] X. H. Hu, X. Sun, Y. Ren, Examination of individual phase textures from HEXRD measurmeent and deformation anisotropy of a QP980 steel., In Preparation (2016).

[31] P. Van Houtte, The MTM-FHM software system, version 2, Katholieke Universiteit Leuven, Belgium, 2000.

[32] H. J. Bunge, Texture in Materials Science: Mathmatical Methods, Butterworth-Heinemann, 1982.

[33] P. Van Houtte, S. Li, M. Seefeldt, L. Delannay, Deformation texture prediction: from the Taylor model to the advanced Lamel model, International Journal of Plasticity 21 (2005), 589-624.

[34] P. Van Houtte, L. De Buyser, The influence of crystallographic texture on diffraction measurements of residual stress, Acta Metallurgica et Materialia 41 (1993), 323-336. 
[35] K. S. Choi, X. H. Hu, X. Sun, M. D. Taylor, E. De Moor, J. Speer, D. K. Matlock, Effects of Constituent Properties on Performance Improvement of a Quenching and Partitioning Steel, SAE TECHNICALPAPER SERIES 2014-01-0812 (2014).

[36] J. W. Hutchinson, Elastic-Plastic Behaviour of Polycrystalline Metals and Composites, Proceedings of the Royal Society of London Series A-Mathematical and Physical Sciences 319 (1970), 247-\&.

[37] J. W. Hutchinson, Bounds and Self-Consistent Estimates for Creep of Polycrystalline Materials, Proceedings of the Royal Society of London Series A-Mathematical and Physical Sciences 348 (1976), 101-127.

[38] P. A. Turner, C. N. Tome, A study of residual stresses in Zircaloy-2 with rod texture, Acta Metallurgica et Materialia 42 (1994), 4143-4153.

[39] J. D. Eshelby, The Determination of the Elastic Field of an Ellipsoidal Inclusion, and Related Problems, Proceedings of the Royal Society of London A: Mathematical, Physical and Engineering Sciences 241 (1957), 376-396.

[40] A. Needleman, R. J. Asaro, J. Lemonds, D. Peirce, Finite-Element Analysis of Crystalline Solids, Computer Methods in Applied Mechanics and Engineering 52 (1985), 689-708.

[41] X. H. Hu, G. A. Cingara, D. S. Wilkinson, M. Jain, P. D. Wu, R. K. Mishra, Studies of Texture Gradients in the Localized Necking Band of AA5754 by EBSD and Microstructure-Based Finite Element Modeling, Cmc-Computers Materials \& Continua 14 (2009), 99-123.

[42] Y. Huang, A user-material subroutine incorporating single crystal plasticity in the ABAQUS finite element program, in , Division of Applied Sciences, Harvard University, Cambridge, MA, 1991.,

[43] J. W. Kysar, Continuum simulations of directional dependence of crack growth along a copper/sapphire bicrystal interface. Part I: experiments and crystal plasticity background, Journal of the Mechanics and Physics of Solids 49 (2001), 1099-1128.

[44] R. A. Lebensohn, C. N. Tome, A self-consistent anisotropic approach for the simulation of plastic deformation and texture development of polycrystals: Application to zirconium alloys, Acta Metallurgica et Materialia 41 (1993), 2611-2624.

[45] N. T. Carlos, Self-consistent polycrystal models: a directional compliance criterion to describe grain interactions, Modelling and Simulation in Materials Science and Engineering 7 (1999), 723

[46] K. Zhang, B. Holmedal, O. S. Hopperstad, S. Dumoulin, J. Gawad, A. Van Bael, P. Van Houtte, Multi-level modelling of mechanical anisotropy of commercial pure aluminium plate: Crystal plasticity models, advanced yield functions and parameter identification, International Journal of Plasticity 66 (2015), 3-30. 

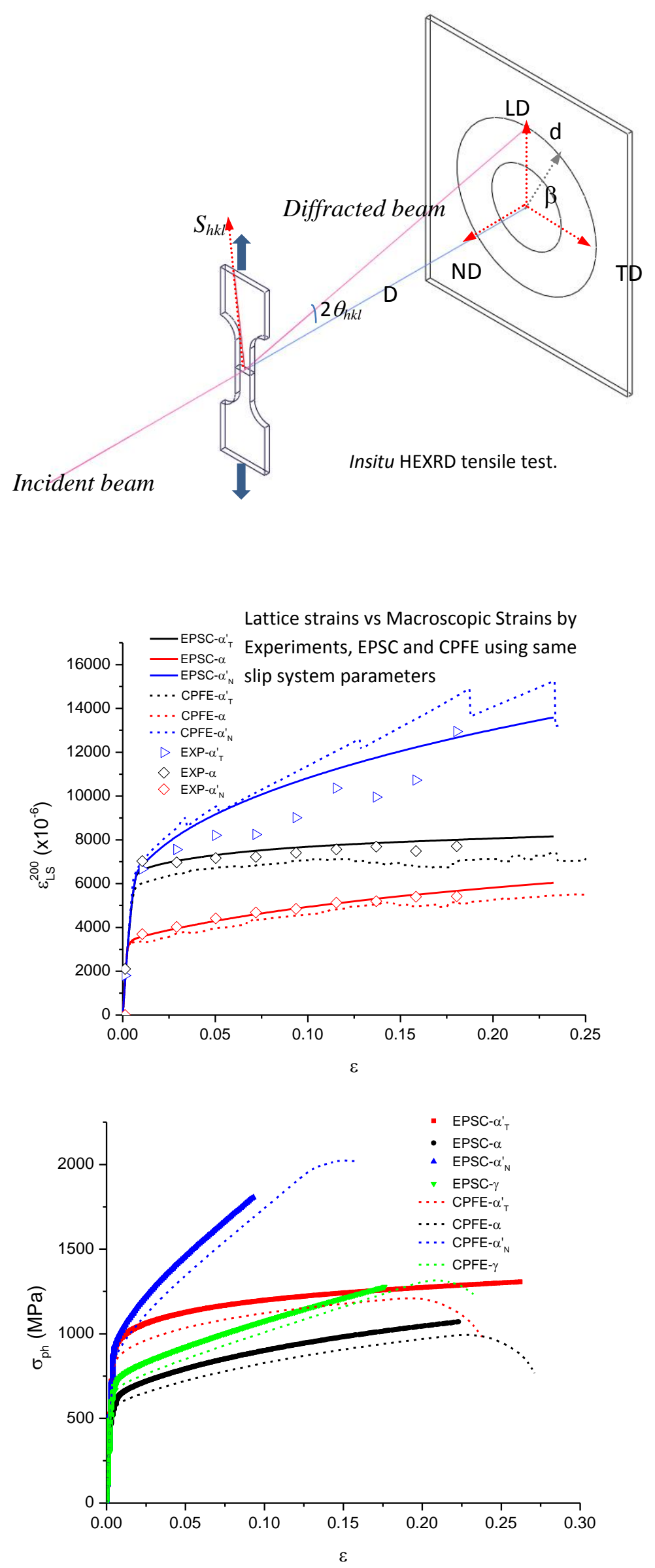

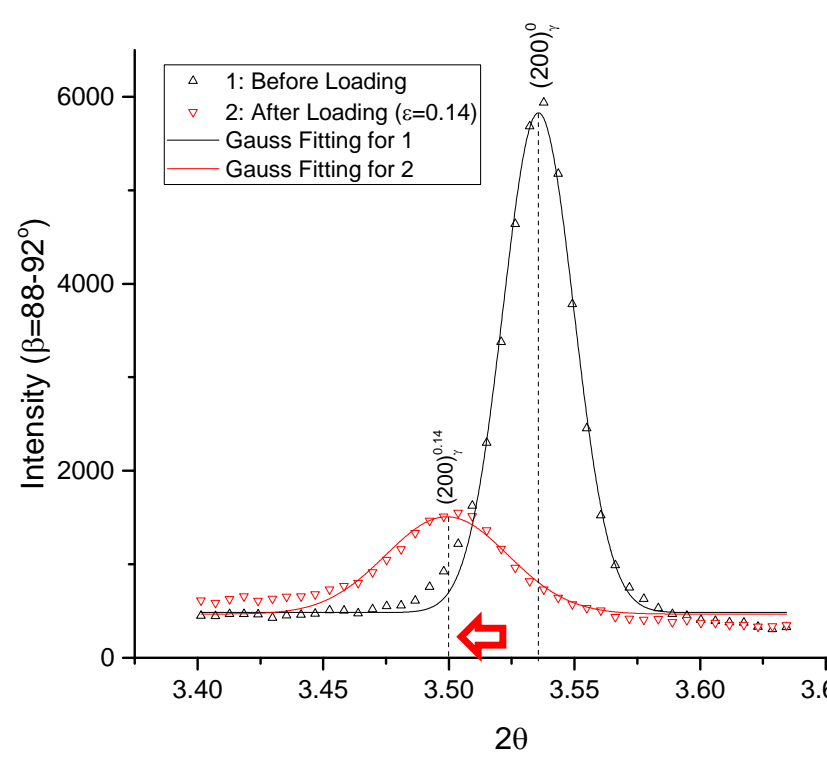

Calculate Lattice Strains by analyzing peak shift

Phase stresses vs Phase Strains by EPSC and CPFE using same slip system parameters 
